\begin{tabular}{|l|l|l|l|l|l|}
\hline MUNIBE Antropologia-Arkeologia & $n^{\circ} 69$ & $123-144$ & DONOSTIA & 2018 & ISSN 1132-2217 • elSSN 2172-4555 \\
\hline
\end{tabular}

\title{
La Cueva de la Dehesilla (Sierra de Cádiz): vuelta a un sitio clave para el Neolítico del sur de la península ibérica
}

\section{Dehesilla Cave (Cádiz Mountains): returning to a key archaeological site for the Neolithic of the Southern Iberian Peninsula}

PALABRAS CLAVES: Neolitización, estratigrafía, cerámica, industria lítica, datación radiocarbónica.

GAKO-HITZAK: eolitizazioa, estratigrafia, zeramika, industria litikoa, datazio erradiokarbonikoa.

KEY WORDS: Neolithization, stratigraphy, pottery, lithic, radiocarbon.

\section{Daniel GARCíA-RIVERO(1,2,3), Juan Carlos VERA-RODRÍGUEZ(4), Manuel Jesús DÍAZ-RODRíGUEZ(1), María BARRERA-CRUZ ${ }^{(1)}$, Ruth TAYLOR ${ }^{(1,2,3)}$, Luis Gethsemaní PÉREZ-AGUILAR ${ }^{(1,3)}$ y Cláudia UMBELINO ${ }^{(2,3)}$}

\section{RESUMEN}

La Cueva de la Dehesilla contó con dos excavaciones arqueológicas en 1977 y 1981. Desde entonces se conoce el potencial prehistórico del sitio, especialmente en relación con sus fases neolíticas, y se convirtió en uno de los enclaves fundamentales en el sur de la península ibérica.

Este artículo presenta los datos procedentes de las recientes intervenciones arqueológicas retomadas cuatro décadas después. Concretamente se da a conocer la secuencia estratigráfica prehistórica de un sondeo efectuado en 2016, sus conjuntos cerámico y lítico, así como cuatro fechas radiocarbónicas. Entre los resultados, destaca la relativa antigüedad de las nuevas dataciones y la presencia de posible ceramica impressa, indicador material de las primeras poblaciones neolíticas en el Mediterráneo occidental.

Los datos obtenidos implican diversos puntos de discusión sobre el origen y la evolución de las sociedades campesinas y ganaderas a niveles regional y peninsular.

\section{LABURPENA}

Dehesillako kobazuloan bi indusketa arkeologiko egin zituzten 1977an eta 1981ean. Ordutik, badakigu tokiak historiaurreko potentzial handia duela, bereziki bere fase neolitikoekin lotuta. Hori dela eta, Iberiar penintsulako hegoaldean oinarrizko gune bihurtu zen.

Artikulu honek lau hamarkada geroago duela gutxi berriro hasi dituzten lan arkeologikoetatik jasotako datuak aurkezten ditu. Zehatzago esanda, 2016an egindako zundaketa baten historiaurreko segida estratigrafikoa, multzo zeramiko eta litikoa, eta lau data erradiokarboniko biltzen ditu. Emaitza horien artean, nabarmendu beharrekoa da datazio berriak nahiko zaharrak direla eta ceramica impressa delakoa topatu dutela ustez. Mendebaldeko Mediterraneoan lehen populazio neolitikoak zeudela erakusten duen adierazle materiala da hori.

Lortutako datuek eskualde eta penintsula mailetako abeltzainen eta nekazarien gizarteek izandako bilakaerari eta jatorriari buruzko eztabaida sorrarazi dute.

\section{ABSTRACT}

Two archaeological excavations were carried out at Dehesilla Cave in 1977 and 1981. The site gained a great scientific interest then, especially in relation to the Neolithic, and it became one of the key archaeological sites in the Southern Iberian Peninsula.

This paper presents new data from the recent archaeological excavations resumed four decades later. Specifically, it provides the data on the Prehistoric stratigraphic sequence documented in 2016, along with the pottery and lithic assemblages, as well as four radiocarbon dates. Of particular interest is the relative antiquity of the new radiocarbon dates and the presence of possible ceramica impressa, a material proxy of the first Neolithic populations in the Western Mediterranean.

These results raise several discussion key points on the origin and the evolution of farming and herding societies in the Southern Iberian Peninsula.

\section{INTRODUCCIÓN}

La Cueva de la Dehesilla se ubica en el sector oriental del término municipal de Jerez de la Frontera (provincia de Cádiz), aunque el núcleo urbano más cercano es Algar. Se emplaza concretamente a $2,5 \mathrm{~km}$ desde la salida norte de esta última población en dirección a
El Bosque por la carretera CA-6107 (Fig. 1). La boca de la cueva (Fig. 2) se localiza en la coordenada UTM (ETRS89, huso 30) X: 264555; Y: 4061867, a una altura de 290 m s. n. m., en la cara sur del Cerro de la Arrayanosa, que alcanza una cota de $464 \mathrm{~m}$. La base del cerro está drenada por el arroyo Don Benito Morena, afluente de la cuenca hidrográfica del río Majaceite.

\footnotetext{
(1) Dpto. Prehistoria y Arqueología, Universidad de Sevilla, C/Doña María de Padilla, s/n, 41004, Sevilla (Spain). Tif.: 954551417. Email: garciarivero@us.es (2) ICArEHB, Universidade do Algarve.

(3) CIAS, Dpto. Ciências da Vida, Universidade de Coimbra

(4) Dpto. Historia, Geografía y Antropología, CIPHCN, Universidad de Huelva.
} 

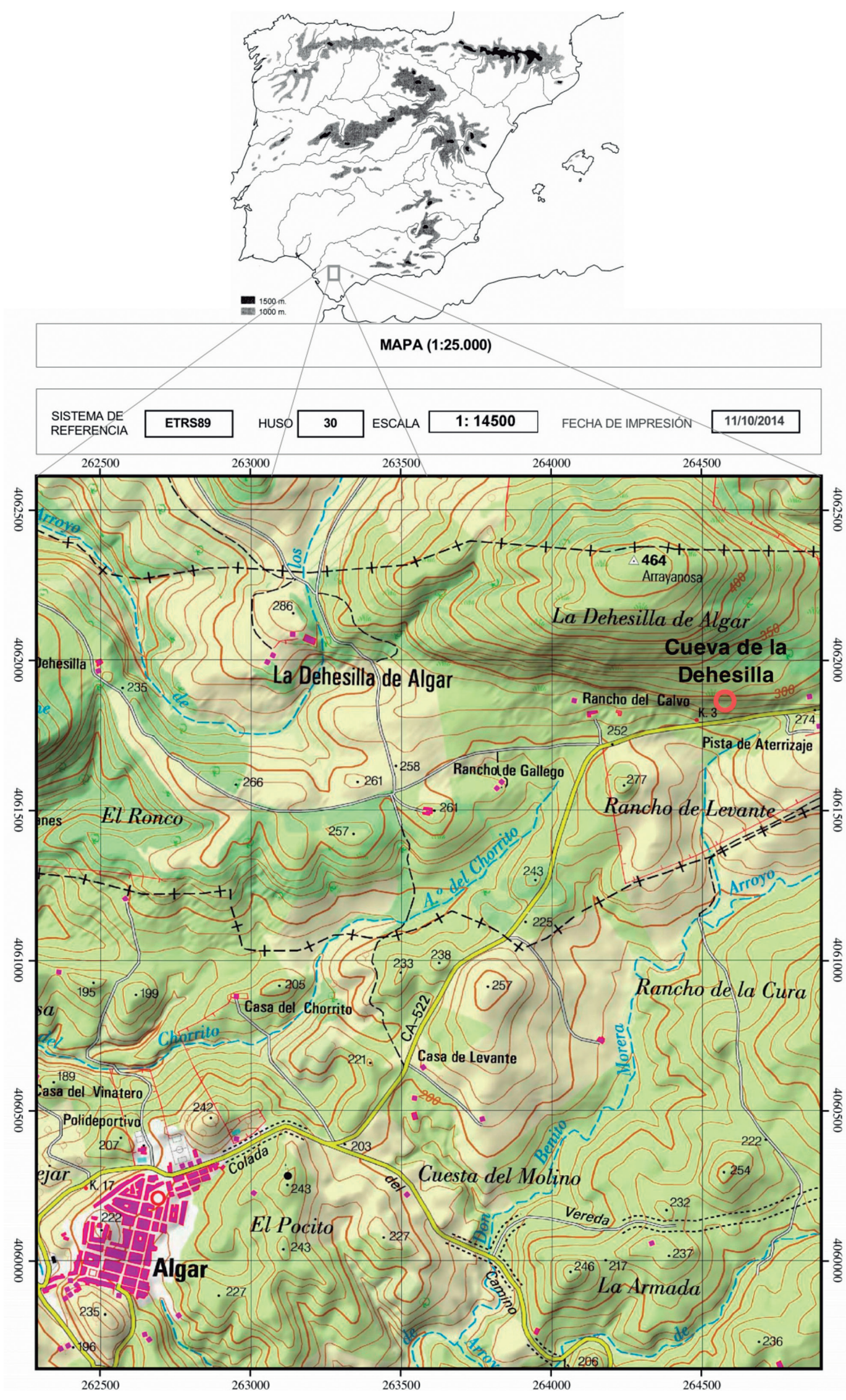

Fig. 1. Ubicación de Cueva de la Dehesilla respecto a la población actual de Algar. A partir del visor IBERPIX, mapa topográfico 1:25.000, Ministerio de Fomento, Gobierno de España. / Location of La Dehesillla Cave in relation to the present-day village of Algar. Based on the IBERPIX viewer, 1:25.000 topographic map, Ministry of Development, Government of Spain. 


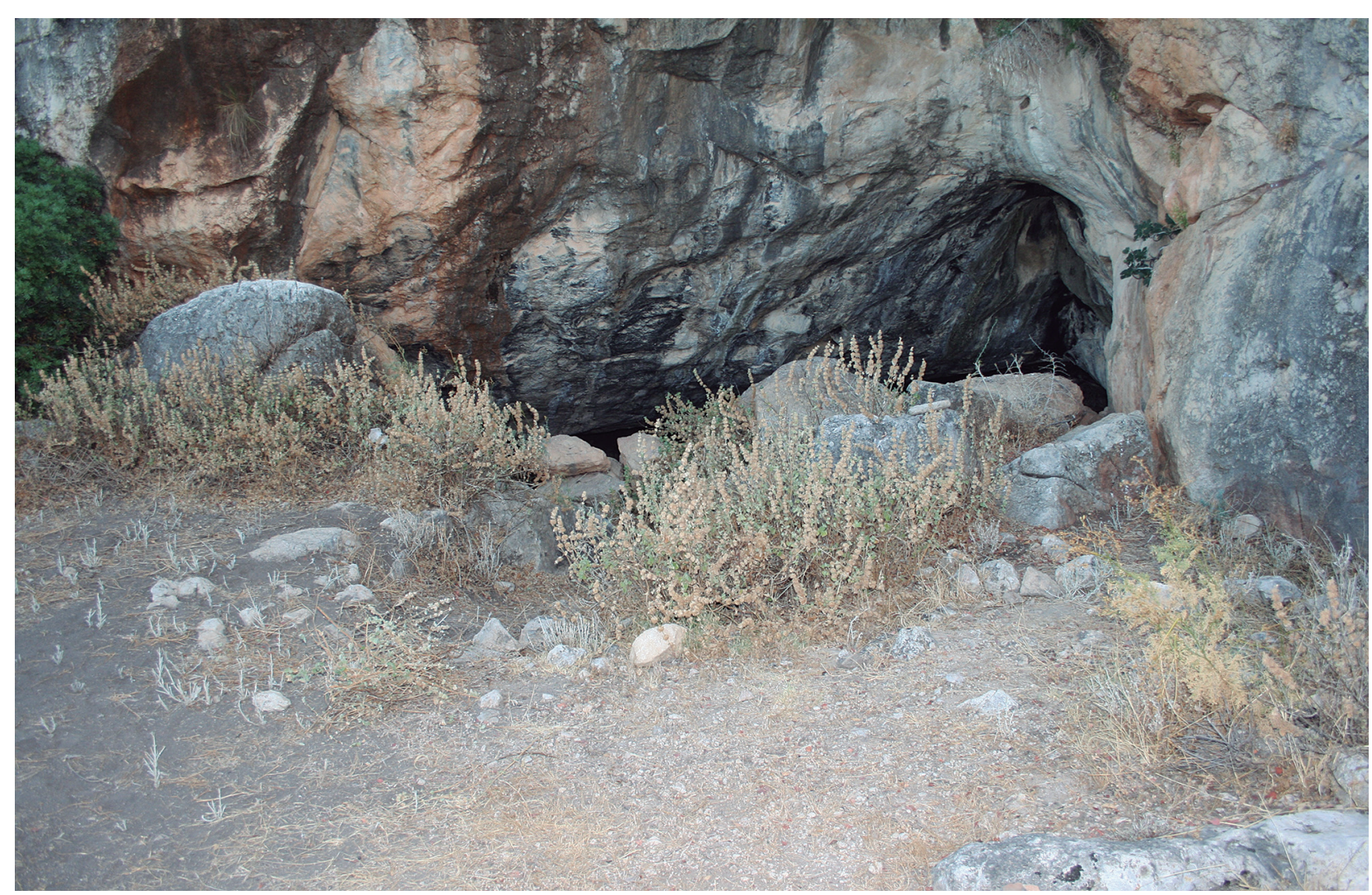

Fig. 2. Boca de acceso a Cueva de la Dehesilla / Entrance of La Dehesilla Cave.

En el complejo geológico de la Sierra de Cádiz se conocen numerosos abrigos y cuevas en los que se ha documentado ocupación antrópica prehistórica. En el entorno próximo de Cueva de la Dehesilla, entre los más conocidos se encuentran las cuevas del Parralejo, en San José del Valle (Pellicer y Acosta, 1982); Cueva de La Pileta (Cortés y Simón, 2007) y Cueva del Gato (Mora-Figueroa, 1976), ambas en Benaoján; Complejo de Veredilla, en Benaocaz (Guerrero-Misa, 1992); la cavidad VR-15, en Villaluenga del Rosario (Santiago et al., 1997); Cueva del Higueral-Guardia (Baena et al., 2012) entre Cortes de la Frontera y Jerez de la Frontera; Cueva del Picado (Mora-Figueroa, 1970) en el último; y la cueva del Higueral de la Valleja (Jennings et al., 2009), en Arcos de la Frontera.

La historiografía arqueológica de la Cueva de la Dehesilla comienza en 1970 (Viñas-Vallverdú, 1970; 1971), cuando un grupo de espeleólogos elaboró un primer mapa topográfico y recogió algunos materiales arqueológicos superficiales y de la limpieza del perfil de un hundimiento. Estos restos se dataron desde el Bronce Final hasta Época Andalusí (Martí et al., 1975).

En 1977 y 1981, los profesores Pilar Acosta y Manuel Pellicer, de la Universidad de Sevilla, realizaron dos cortes en la primera sala (situada a la entrada) mediante los que se documentó una secuencia de ocupación con dos estratos $-\mathrm{VI}$ y $\mathrm{V}$ - considerados como
Neolítico antiguo $A$ y $B$, respectivamente; dos estratos -IVA y IVB- pensados como Neolítico medio A y B, respectivamente; un estrato -III- datado en el Neolítico reciente; y dos estratos - II y I- pertenecientes a la Edad del Cobre (Acosta y Pellicer, 1990). Presentaron resultados de análisis de radiocarbono, que para el estrato inferior de Neolítico antiguo proporcionaron entonces fechas más antiguas de lo esperado para las primeras poblaciones campesinas y ganaderas de la península ibérica (Pellicer y Acosta, 1982; Acosta y Pellicer, 1990: 87 y ss.). Estas fechas generaron bastante controversia acto seguido a su publicación (Fortea y Martí, 1985; Zilhão, 1993; Mederos, 1996; Bernabeu et al., 2001). Los arqueólogos dejaron de excavar cuando alcanzaron un nivel de bloques de roca caliza, y se mostraron entonces cautelosos en la predicción de la existencia de niveles anteriores, epipaleolíticos y/o paleolíticos (Acosta y Pellicer, 1990: 27).

Las estratigrafías presentadas por Acosta y Pellicer fueron en buena medida incomprendidas, rechazadas o ignoradas a lo largo de más de tres décadas, debido a la combinación de diversos factores, el principal de los cuales se relacionaba con los resultados y el tratamiento de las fechas absolutas obtenidas (Tabla I). De las siete muestras analizadas, seis fueron determinadas por el laboratorio Gakushuin (GaK) de Tokyo (Japón), y la restante por el de la Universidad de Granada (UGRA) (Acosta y Pellicer, 1990). Este hecho ha venido 


\begin{tabular}{|c|c|l|c|c|c|}
\hline Código & ID & \multicolumn{1}{|c|}{ Contexto } & Muestra & Fecha BP & Cal BC - 2 $\boldsymbol{~}$ \\
\hline GaK 8956 & 10 & DE-77, Estrato IV-B, Nivel 8, NM-B & Carbón & $5920 \pm 170$ & $5224-4375$ \\
\hline GaK 8957 & 11 & DE-77, Estrato IV-B, Nivel 8, NM-B & Carbón & $8200 \pm 160$ & $7548-6707$ \\
\hline GaK 8958 & 12 & DE-77, Estrato IV-A, Nivel 10, NM-A & Homo sapiens & $3120 \pm 180$ & $1865-905$ \\
\hline GaK 8955 & 9 & DE-77, Estrato IV-A, Nivel 10, NM-A & Carbón & $7040 \pm 170$ & $6239-5624$ \\
\hline GaK 8954 & 8 & DE-77, Estrato IV-A, Nivel 10, NM-A & Carbón & $7120 \pm 200$ & $6391-5651$ \\
\hline GAK 8953 & 7 & DE-77, Estrato V, Nivel 11, NA-B & Carbón & $7670 \pm 400$ & $7513-5804$ \\
\hline UGRA 259 & 1 & DE-81, Estrato VI, Nivel 13, NA-A & Carbón & $6260 \pm 100$ & $5468-4992$ \\
\hline
\end{tabular}

Tabla 1: Dataciones disponibles ordenadas según la secuencia propuesta (Acosta y Pellicer, 1990: 87). Calibración efectuada con Calib 7.0 (Reimer et al., 2013). / Available dates ordered according to the proposed sequence (Acosta and Pellicer, 1990: 87). Calibration performed with Calib 7.0 (Reimer et al., 2013).

lastrando hasta la actualidad los debates cronológicos relativos a la secuencia de la cavidad, al centrarse las discusiones en la significancia de la serie nipona. A este respecto cabe destacar que ya desde la década de 1980 se vino comprobando en diversos yacimientos cómo los resultados proporcionados por GaK adolecían sistemáticamente de un significativo envejecimiento. Así, en aquellos yacimientos en los que una misma muestra contextual fue dividida y enviada tanto a GaK como a otras instituciones diferentes, caso de los Castros pontevedreses de la Edad del Hierro de Penalha y de Troña (Carballo y Fábregas, 1991) contrastados respectivamente con los laboratorios de Groningen (GrN) y del CSIC, las distorsiones son más que evidentes y siempre en la misma dirección ${ }^{1}$. Otros ejemplos evidentes de este fenómeno han sido corroborados posteriormente en yacimientos más cercanos en el tiempo y en el espacio al que nos ocupa, caso de la Cueva de Nerja, dándose la circunstancia de que fueron igualmente investigados por el propio equipo de Acosta y Pellicer, y cuyos análisis de $\mathrm{C} 14$ fueron realizados de manera correlativa a los de Cueva de la Dehesilla en GaK, como lo atestiguan los propios códigos del laboratorio (Pellicer y Acosta, 1997). El tratamiento conjunto, calibrado y estadístico del total de las setenta dataciones radiocarbónicas -procedentes de hasta ocho laboratorios diferentes- disponibles hasta hace una década, ha demostrado la existencia de un anormal envejecimiento que afecta a las datas de GaK, claramente representado en la curva de probabilidad acumulada tanto para la secuencia magdaleniense como para la neolítica (Jordá y Aura, 2008: 251 y 252).

A esta problemática hay que sumar, junto a otros factores como el posible efecto de la "madera vieja", las elevadas desviaciones estadísticas de las dataciones GaK 8953 a 8958 de Cueva de la Dehesilla, que oscilan entre \pm 160 y \pm 400 años, y una circunstancia adicional, que enmarañó aún más las disquisiciones crono-estratigráficas de la cavidad y que, a la postre, conllevó la incomprensión generalizada de los resultados. En la monografía de 1990, todas las determinaciones BP proporcionadas por GaK fueron presentadas en formato a.C., es decir, sustrayendo directamente 1950 años a la fecha central bruta BP (práctica habitual por los años de la publicación e incluso hasta inicios de los años 90), mientras que el resultado de UGRA 259 lo fue tanto en BP como calibrada por dendrocronología (Acosta y Pellicer, 1990: 87), propiciando nuevas incoherencias en cadena.

No obstante, si seguimos secuencialmente en orden ascendente las dataciones calibradas, según estratos, niveles y fases culturales propuestos en 1990 (Tabla I), las identificadas con los códigos GaK 8953, 8954 y 8955 mantienen una sucesión cronológica descendente, y abarcarían el lapso de la segunda mitad del $\mathrm{VI}$ milenio o inicios del V milenio cal BC si se sustrajera la media de 700 años a un milenio que calculamos más arriba (véase la nota anterior a pie de página). A éstas les siguen las dos dataciones que hasta la fecha han constituido el principal caballo de batalla a la hora de enfatizar las incongruencias estratigráficas de Cueva de la Dehesilla (Zilhao, 1993; Mederos, 1996). La primera, GaK 8958, realizada sobre hueso de un enterramiento "incrustado" en el nivel 11 pero atribuido al nivel 10, podría corresponderse efectivamente con una inhumación de la Edad del Bronce o del Hierro, a juzgar por su fecha (1865-905 cal BC). Respecto a la segunda, GaK 8957 (8200 \pm 160 BP), hemos detectado una contradicción en la presentación de las fechas brutas en publicaciones posteriores a la monografía de 1990, donde, identificada con idéntico código de laboratorio, la fecha aparece consignada como 7440 160 BP (Acosta, 1995: 36; Pellicer y Acosta, 1997: 362). Si eso fuese así, y la data pu-

\footnotetext{
1 En el primer caso, sendas determinaciones sobre una misma muestra de carbón vegetal proporcionaron unas fechas oscilantes entre $2485 \pm 35$ BP (GrN-14132) y 4010 120 BP (GaK-12970), es decir, 1525 años de diferencia entre las fechas centrales. Otras realizadas sobre muestras

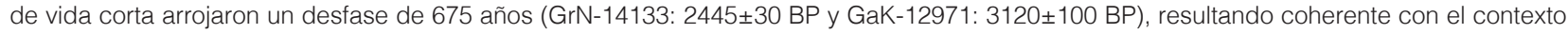
arqueológico tan solo la más reciente del laboratorio holandés (Carballo y Fábregas, 1991: 254). En el segundo, hasta tres determinaciones de contraste sobre las mismas muestras de madera carbonizada (CSIC-688: 2230 \pm 50 BP vs GaK-12222: 2960 \pm 120 BP; CSIC-689: $2330 \pm 50$ BP vs GaK-12223: 3100 \pm 90 BP, y CSIC-690: 2400 \pm 50 BP vs GaK-12224: 3470 110 BP) constataron respectivamente significativas diferencias de 730, 770 y 1070 años entre el laboratorio español y el japonés (Carballo y Fábregas, 1991: 256). Tomadas en conjunto, las cinco dataciones arrojan una media de 954 años de mayor antigüedad, es decir, casi un milenio de desajuste, con una mínima que ronda los 700 años.
} 
blicada en 1990 fuese la errónea, su corrección y calibración a dos sigmas se superpondría ampliamente con las oscilaciones del resto de las determinaciones sobre carbón citadas anteriormente, llegando en su límite inferior hasta inicios del V milenio. Por último, la datación más reciente, GaK 8956, se situaría entre la segunda mitad del $\mathrm{V}$ y el primer tercio del IV milenios cal BC si aplicásemos el mismo criterio correctivo anterior.

En cualquier caso, cabe tomar en consideración que Cueva de la Dehesilla contaba no obstante con una determinación (UGRA 259: $6260 \pm 100$ BP) (González et al., 1987: 385) perfectamente coherente con los datos conocidos ya desde la década de los 70 hasta la actualidad para el neolítico meridional, y no afectada por la casuística que venimos comentando. Procedente del nivel más antiguo (Nivel 13) del estrato basal (Estrato VI) de 1981, correspondiente al denominado entonces Neolítico Antiguo A, su calibración a dos sigmas (5468-4992 cal BC) era y es considerada representativa (e.g. Martín-Socas et al., 2018) de un Neolítico antiguo andaluz con cerámicas a la almagra coincidente cronológicamente con las dataciones antiguas y más recientes de los principales yacimientos andaluces en cueva, como Nerja (Pellicer y Acosta, 1997; Jordá y Aura, 2008; García Borja et al., 2010, 2014), Murciélagos de Zuheros (Vicent y Muñoz, 1973; Vera-Rodríguez y Gavilán, 1999; Peña-Chocarro et al., 2013), del Toro (Martín-Socas et al., 2004; Égüez et al., 2014) y Carigüela (Fernández et al., 2007; Medved, 2013).

Dado el interés científico del sitio, en 2015 se inició el Proyecto General de Investigación Cueva de la Dehesilla: Estudio arqueológico y paleoambiental para el conocimiento de la ocupación humana prehistórica de la Sierra de Cádiz, dirigido por el primer firmante de este trabajo (DGR). Este mismo año, se realizó una reconstrucción topográfica tridimensional de la cavidad y asimismo se iniciaron los estudios geomorfológicos y geoarqueológicos (García-Rivero et al., en prensa, a y b). Durante los meses de agosto y septiembre de 2016 se ha desarrollado la primera excavación arqueológica del proyecto, que ha constituido la ejecución de dos cortes en la terraza (exterior) frente a la boca de la cueva, así como un sondeo en su interior (Fig. 3). Los estratos propiamente prehistóricos se encuentran en el interior de la cavidad, ya que en los cortes externos -aun conteniendo escasos restos prehistóricos en niveles históricos- no se han encontrado evidencias directas de ocupación prehistórica sino islámica (Taylor et al., 2018; García-Rivero et al., en prensa, c). Las analíticas de numerosas muestras arqueológicas de variada naturaleza siguen en proceso de estudio.

El objetivo fundamental del presente trabajo es dar a conocer los primeros datos inéditos procedentes de la secuencia estratigráfica prehistórica documentada en 2016 en el sondeo interno, denominado C003. Se describen las características sedimentológicas principales de cada fase estratigráfica. También se presentan sus conjuntos cerámicos y líticos, cuyo análisis permite

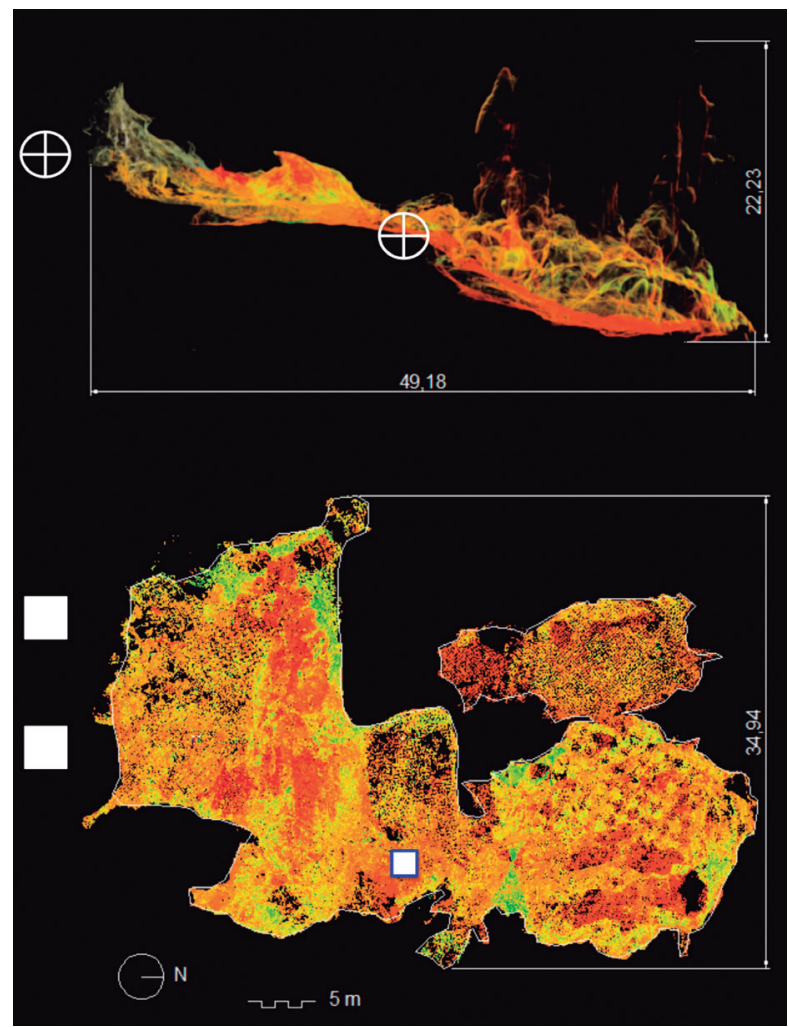

Fig. 3. Ubicación de los dos cortes y sondeo estratigráficos en la sección longitudinal y planta del modelo topográfico 3D. A partir de García-Rivero et al., en prensa, b, Fig. 7). Los ubicados exteriormente frente a la boca de la cavidad, C001 y C002, presentan una secuencia andalusí; el sondeo ubicado en el interior -cuyos bordes se resaltan en color azul- se denomina C003, con una secuencia prehistórica e histórica. / Location of the two excavation areas and the stratigraphic test pit on the longitudinal section and the 3D topographic model. After García-Rivero et al., in press b. Fig. 7). The areas outside the cave entrance, C001 and C002, yielded an Andalusi sequence; the area inside the cave, $\mathrm{C003}$, marked in blue, provided a Prehistoric and Historical sequence.

identificar y caracterizar las distintas facies culturales y cronológicas. Esta cultura material se establece como indicadora de las ocupaciones - del consumo y desechos humanos- de cada una de estas facies, sin que, por el momento, dado el tamaño del sondeo, pueda saberse si responden a deposiciones de carácter primario. No obstante, sí existen evidencias en este último sentido, como las unidades $12 \mathrm{~b}$ y Hogar 1. También se dan a conocer asimismo los resultados de los análisis radiocarbónicos efectuados, los cuales ayudan a precisar la cronología de los distintos episodios estratigráficos y culturales de la secuencia neolítica. Por último, se realiza una discusión sobre la información anteriormente conocida de este asentamiento a la luz de los nuevos datos, especialmente en términos cronológicos y particularmente respecto a la definición de los diferentes periodos arqueológicos neolíticos del sitio. Asimismo, se ponen en relación cada uno de estos episodios culturales documentados en Cueva de la Dehesilla con el poblamiento prehistórico conocido actualmente en el contexto geográfico regional próximo, y se indican las contribuciones más relevantes del estudio respecto al marco del sur peninsular. 


\section{TÉCNICAS Y MÉTODOS}

La ubicación y las dimensiones del sondeo C003 vinieron dadas por la localización de una afección clandestina que hubo de producirse entre el 5 de diciembre de 2015 y el 12 de febrero de 2016, la cual se notificó rápida y debidamente a las autoridades competentes de la Dirección General de Bienes Culturales y Museos de la Junta de Andalucía. El daño observado constaba de una abertura de forma circular de en torno a 1,9 m de diámetro y 1,8 m de profundidad (posteriormente sabríamos que afectó a los niveles históricos y parcialmente a uno existente de la Edad del Cobre, pero no alcanzó el estrato superior de la secuencia neolítica). Autorizado oficialmente por la administración, se planteó un sondeo rectangular de 1,8 m en dirección N-S y de 1,5 m en dirección E-O. Los vértices del planteamiento de dicho rectángulo tienen las siguientes coordenadas UTM (ETRS89, huso 30: 1) X: 264562.182015, Y: 4061892.00200, Z: 279.689; 2) X: 264563.700020, Y: 4061891.955004, Z: 279.648; 3) X: 264563.721993, Y: 4061890.147021, Z: 279.935; y 4) X: 264562.206978, Y: 4061890.173021, Z: 279.861. Dada las reducidas dimensiones del sondeo, se juzgó conveniente no entibar los perfiles, pero sí el conferirles una ligera inclinación que minimizase cualquier riesgo de desprendimiento. Por razones de seguridad, se decidió no actuar sobre el perfil $O$, al ser éste el lado por el cual se accedía al interior del sondeo mediante el uso de escalera. Al final de la intervención, el sondeo alcanzaba una profundidad en torno a 4,70 m (siendo la cota $+275,235$ $\mathrm{m})$, y su base tenía unas dimensiones aproximadas de $0,7 \times 0,5 \mathrm{~m}$. Dada su elevada profundidad y reducidas dimensiones espaciales, así como la gran cantidad de bloques calizos en el estrato inferior, que alimentaban la probabilidad de riesgo de desprendimientos, no se llegó a agotar la secuencia arqueológica del área.

La excavación se produjo de manera sumamente cuidadosa atendiendo a la definición de las interfaces de las unidades estratigráficas (Harris, 1991), de forma que todo el material y muestras arqueológicas quedaron debidamente registrados en sus correspondientes estratos. En todos éstos se cribó mecánicamente el $50 \%$ de la tierra extraída, mientras que el 50\% restante se flotó. El material cerámico procedente de cada estrato se ha pesado y contabilizado en función de distintas categorías (número total, fragmentos con información morfométrica o decorativa, número y porcentajes de cerámica decorada). Las categorías decorativas son acanalada, engobe a la almagra, excisa, grabada, impresión, incisión, aplicación plástica y peinada. Las cuantificaciones absolutas y porcentuales que se presentadan hacen referencia al número de fragmentos, y no a una estimación del número de recipientes, aunque sí se han tratado unitariamente los fragmentos que conectan físicamente. En el apartado Resultados se aportan los dibujos de una selección que representa apropiadamente en términos morfométricos y decorativos la diversidad del conjunto de cada estrato.
La industria lítica se ha cuantificado y estudiado atendiendo a dos grandes apartados: industria lítica tallada y piedra pulimentada. El estudio de las técnicas de talla lítica se ha basado en la tipología analítica de Laplace (1966) en lo referente al retoque y a determinadas técnicas de extracción, completando la definición de determinados atributos técnicos mediante la metodología de Bernaldo-Quirós et al. (1981). Por su parte, la tipometría del material ha sido establecida siguiendo la propuesta de Bagolini (1968). Con carácter general, la industria lítica se ha clasificado en Material Retocado o Material Sin Retocar. El primer apartado comprende los útiles, hojas y lascas retocadas y los varios retocados, mientras el segundo se compone de hojas, lascas, informes, núcleos, restos de núcleo y productos de acondicionamiento y chunks. La presencia de embotamientos, patinaciones y huellas de uso macroscópicas han sido determinadas mediante la observación de los filos con una lupa x20. El análisis tecno-tipológico se ha completado metodológicamente considerando las principales síntesis y propuestas de léxico elaboradas para las zonas occidental (Carvalho, 1998; Carvalho y Gibaja, 2005) meridional (Martínez y Afonso, 2008) y oriental (Juan-Cabanilles, 2008) de la península ibérica, si bien realizando las adaptaciones oportunas a las particularidades de Cueva de la Dehesilla.

\section{RESULTADOS}

\subsection{Secuencia estratigráfica del sondeo $\mathrm{COO3}$}

El sondeo C003 permite una aproximación a la secuencia estratigráfica de un sector intermedio del interior de la cueva (Figs. 3, 4 y 5). El sondeo ha permitido documentar 25 unidades estratigráficas (UE) cuya nomenclatura va desde UE 1 a UE 16b (Fig. 6). A la espera de los resultados de los análisis microestratigráficos y sedimentológicos, pueden sugerirse preliminarmente, a partir de las características sedimentológicas de visu, 6 grandes fases estratigráficas (cada una, a su vez, conformadas por diversas UUEE). Como veremos, las 4 fases inferiores son prehistóricas. De ellas, la superior es calcolítica y las tres inferiores neolíticas, siendo éstas las que consideramos en este trabajo y detallamos a continuación.

Desde la parte inferior a la superior de la secuencia, la primera fase estratigráfica está conformada a su vez por las unidades estratigráficas 16b y 16a. UE 16b tiene una matriz sedimentaria de color marrón anaranjado y alta presencia de pequeñas raíces y carbón. Su textura es arenosa. Está compuesta por una gran cantidad de grandes y medianos bloques de piedra endógena y calizas blanquecinas, cuyas dimensiones máximas pueden alcanzar los $30 \mathrm{~cm}$. En su base aparecen algunos bloques impregnados de arcilla roja entre las que se filtra el sedimento descrito. Por otro lado, UE 16a se refiere a un estrato de color marrón oscuro, de textura igualmente arenosa, con abundantes carbo- 


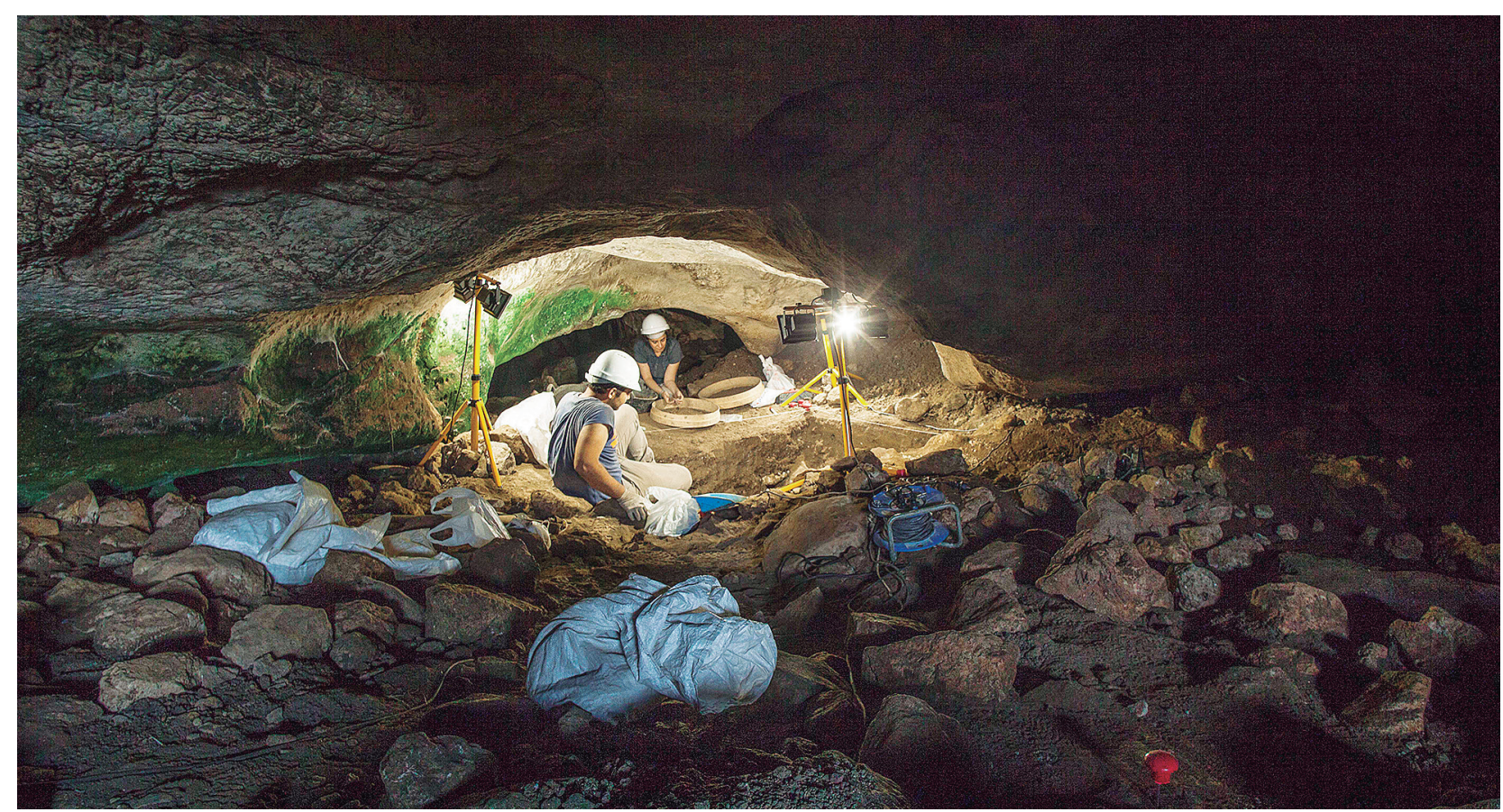

Fig. 4. Fotografía del entorno inmediato, y del proceso de excavación, del sondeo C003. (Autor: Javier Pérez González, Proyecto Dehesilla). / Photograph o the immediate surroundings and the excavation process of C003. (Photo: Javier Pérez González, Proyecto Dehesilla).

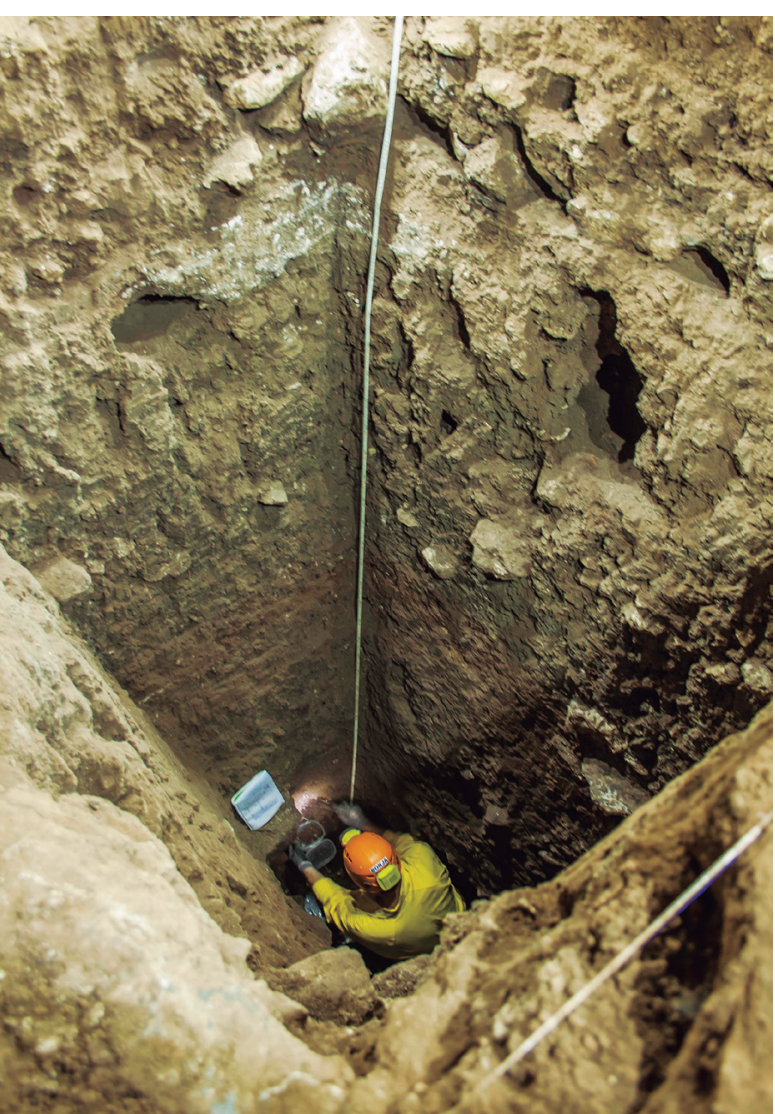

Fig. 5. Fotografía de los perfiles $N$ (izquierda) y $E$ (derecha) del sondeo C003 durante la extracción de muestras analíticas. / Photograph of the North (left) and East (right) sections of C003 during the extraction of samples for analysis.

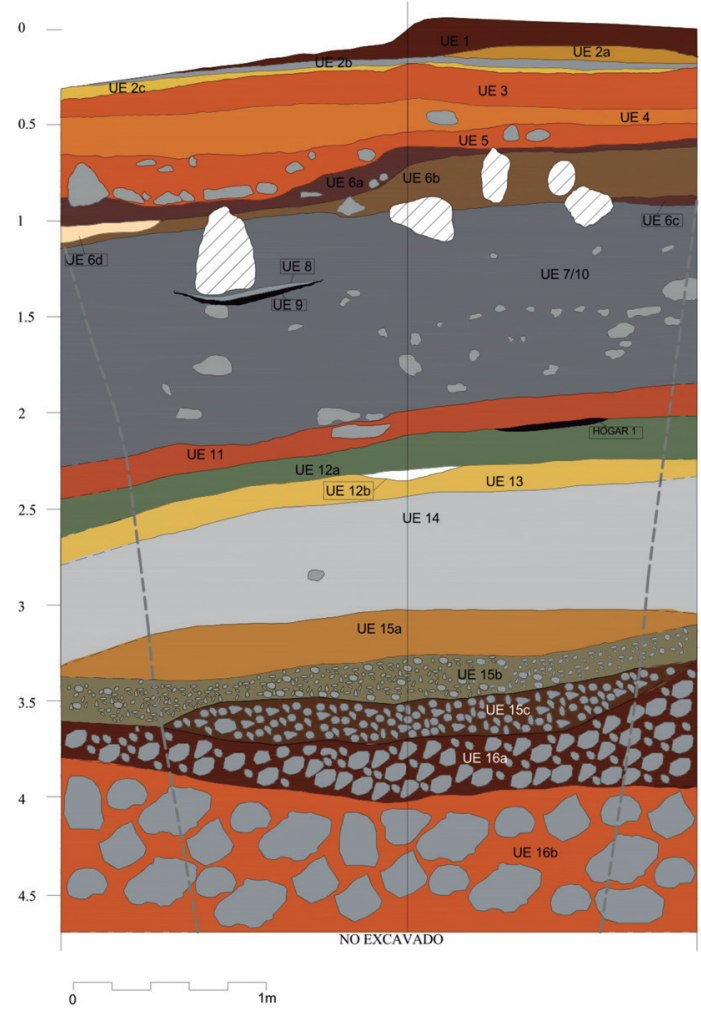

Fig. 6. Perfiles Este (izquierda) y Sur (derecha) del sondeo C003. Por medidas de seguridad, los perfiles se excavaron confiriéndoles una ligera pendiente conforme se profundizaba (nótense las líneas discontinuas). / East (left) and South (right) sections of C003. For safety reasons the sides of the test pit were slightly sloped towards the base (as indicated by the dashed lines). The estimation of the stratigraphic contacts in the areas are shown here (also in dashed lines) assuming their known inclination. 
nes y pequeñas raíces. Tiene presencia igualmente de clastos endógenos de entre 5 y $15 \mathrm{~cm}$, diferenciándose de la UE 16b por el menor tamaño y densidad de éstos.

La segunda fase estratigráfica agrupa a las UUEE $15 \mathrm{c}, 15 \mathrm{~b}$ y posiblemente 15a. En primer lugar, UE 15c es un estrato de color marrón oscuro, bastante arcilloso y compacto, con bloques de tamaño mediano -en torno a 15 cm-, pequeñas raíces y carbón. Además, tiene nódulos calizos y gravas de pequeño y mediano tamaño. Se le superpone UE 15b, que es de color marrón grisáceo, también con textura arcillosa y estructura compacta. Alberga gran cantidad de carbones y de clastos endógenos. Por último, UE 15 a es un estrato muy arcilloso y compacto, de un color marrón beige, con abundantes fragmentos de carbón, pero con apenas clastos, que aparecen de forma esporádica, y sin raíces.

La tercera fase estratigráfica comprende a las UUEE 14, 13, 12b, 12a, Hogar 1 y 11. En primer lugar, UE 14 es un estrato de cierta potencia, pero de deposición lenta y composición homogénea. Es de color gris, de textura arcillosa, con tendencia a formar nódulos, y de estructura compacta. Presenta mucha materia orgánica. Se le superpone UE 13, que es de color amarillento con motas rojas y muchos trozos de carbón; igualmente tiene una textura arcillosa y una estructura muy compacta. Sobre su interface superior se encuentra UE $12 b$, concretamente en la esquina de los perfiles Sur y Este. Se trata de una pequeña capa arcillosa de color blanquecino con fracciones de carbón. Se sobrepone a ambas UE 12a, un estrato de deposición lenta y composición homogénea. Tiene un color gris-amarillento y presenta gran concentración de carbones. Es de textura arcillosa y compacta, con escasos nódulos de caliza pequeños. Se ha denominado Hogar 1 a una fina capa de cenizas localizada sobre la interfaz superior de UE 12a. Finalmente, el estrato UE 11 es de color marrón claro, con tonalidad anaranjada. De deposición lenta y composición homogénea, se trata de un estrato de textura arcillosa y compacto, con menos restos de carbón que UE $12 \mathrm{a}$.

\subsection{Conjunto cerámico}

El conjunto de cerámica documentado en las unidades neolíticas del sondeo C003 es aproximadamente de $10 \mathrm{~kg}$ (Tabla II). A nivel general, cuatro estratos destacan con creces en cuanto al peso y cantidad de cerámica, si bien sus posiciones relativas varían sensiblemente según ambos parámetros. Los estratos que destacan en cuanto a mayor peso cerámico son ordinalmente 15c, 14, 15a y 11, mientras que el orden según la cantidad de fragmentos es $14,11,15 a$ y $15 \mathrm{c}$. Los fragmentos que permiten información tipológica o decorativa (Tabla II) son más frecuentes en los estratos $14,15 a, 15 c, 11,15 b$ y $12 a$.

En relación exclusivamente con la cerámica decorada, los estratos con más cantidad son, a mucha distancia del resto, $15 a$ y 14 , seguidos de los niveles $15 \mathrm{c}$ y 15b (Tabla II y Fig. 7A). Sin embargo, si se atiende al porcentaje decorado del conjunto de cada estrato se ubican en primer lugar 16a y 15b, seguidos de $15 a$ y 15c. Si atendemos a la diversidad de la técnica decorativa (Fig. 7B), predominan en gran parte de la secuencia las cerámicas incisas e impresas. Se produce una diversificación de las técnicas decorativas desde el estrato $15 \mathrm{c}$ hasta la unidad 13, en cuyo intervalo están representadas de mayor a menor el engobe de almagra, la incisión, la impresión, la grabada o incisión en seco, las aplicaciones plásticas (de mamelones o cordones) y finalmente la excisa y la acanalada. La acanalada sólo aparece en la unidad 15b, la excisa sólo en los estratos $15 a$ y 14 , la grabada sólo en la unidad 14 y finalmente la peinada sólo en el estrato 12b. No obstante, si se atiende al porcentaje decorado del conjunto de cada estrato se ubican en primer lugar 16a y 15b, seguidos de $15 a$ y $15 c$

Estas diferencias entre las frecuencias de cerámica relevante en términos morfométricos y decorativos a lo largo de la estratigrafía parecen no explicarse momentáneamente en función sólo del espesor y volumen de los estratos. Las analíticas sedimentológicas permitirán

\begin{tabular}{|c|c|c|c|c|c|c|}
\hline Nombre estratos & Peso (g) & $\mathrm{N}^{\circ}$ total fragm. & $\mathrm{N}^{\circ}$ fragm. sin forma/decor & $\mathrm{N}^{\circ}$ fragm. con forma/decor & $\mathrm{N}^{\circ}$ fragm. con decor & $\%$ decor \\
\hline UE 11 & 1071 & 235 & 214 & 21 & 6 & 2,55 \\
\hline Hogar 1 & 148 & 24 & 22 & 2 & 0 & 0 \\
\hline UE 12A & 480 & 98 & 84 & 14 & 9 & 9,18 \\
\hline UE 12B & 88 & 13 & 12 & 1 & 1 & 7,62 \\
\hline UE 13 & 130 & 28 & 22 & 6 & 5 & 17,85 \\
\hline UE 14 & 1614 & 344 & 269 & 75 & 45 & 13,08 \\
\hline UE $15 \mathrm{~A}$ & 1268 & 174 & 113 & 61 & 51 & 29,31 \\
\hline UE 15B & 409 & 34 & 17 & 17 & 15 & 44,11 \\
\hline UE 15C & 2014 & 115 & 81 & 34 & 24 & 20,17 \\
\hline UE $16 \mathrm{~A}$ & 61 & 8 & 4 & 4 & 4 & 50 \\
\hline UE 16B & 18 & 2 & 2 & 0 & 0 & 0 \\
\hline
\end{tabular}

Tabla 2: Conjunto cerámico procedente de los niveles neolíticos del corte C003./ Pottery assemblage from the Neolithic levels of area C003. 

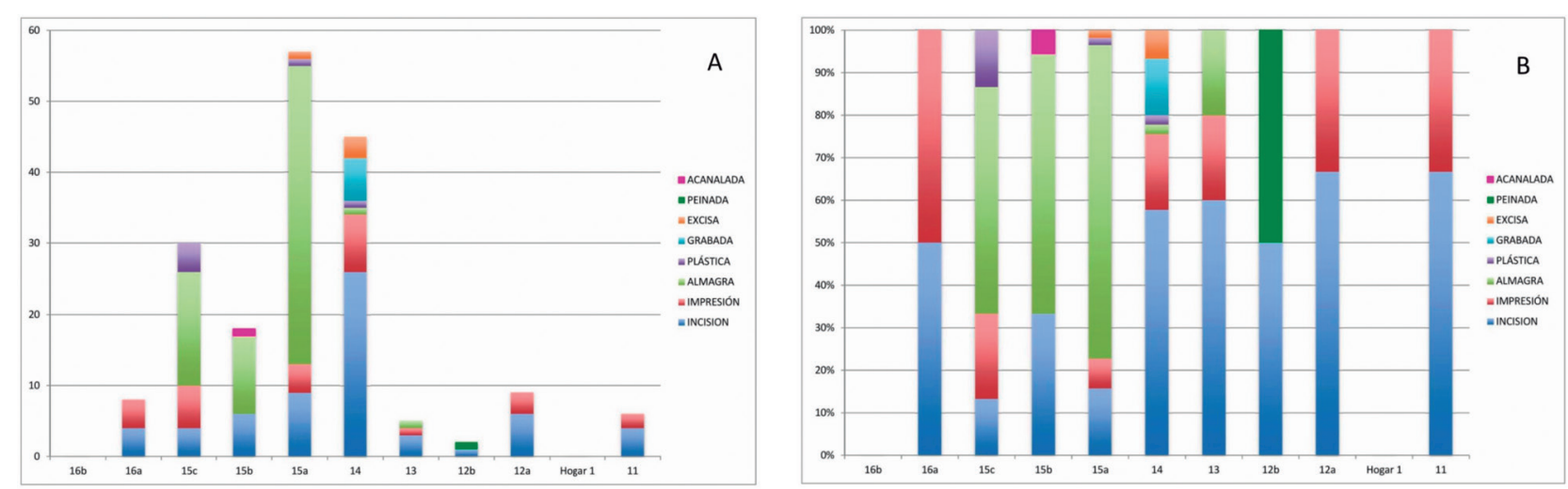

Fig. 7. A) Gráfico de barras apilado con las frecuencias relativas de los diferentes géneros de decoración cerámica; y B) Gráfico de barras apilado con los porcentajes relativos de los distintos géneros decorativos. / A) Accumulative bar chart of the relative frequencies of the different decorated pottery types; B) Accumulative bar chart of the percentage of the different decorated pottery types.

información relevante sobre la formación de los depósitos. Por momento, puede pensarse que aquellas diferencias entre algunos de los estratos pueda deberse a posibles patrones temporales y culturales disímiles.

Los estratos inferiores 16b y $16 \mathrm{a}$, correspondientes a la primera fase estratigráfica señalada en el apartado anterior, cuentan con relativa potencia, y albergan una gran cantidad de nódulos y piedras calizas. El inferior y más potente de ellos, 16b, sólo dispone de 2 fragmentos cerámicos, ninguno con información tipológica o decorativa; el segundo en cambio, 16a, cuenta con el porcentaje más elevado de cerámicas decoradas. Aunque su conjunto cerámico total está constituido sólo por 8 fragmentos de pequeño tamaño, es interesante anotar que los 4 fragmentos decorados muestran cierta variedad técnica (Figs. 8 y 9). Cuentan con incisiones e impresiones. Éstas muestran distintas matrices, realiza-
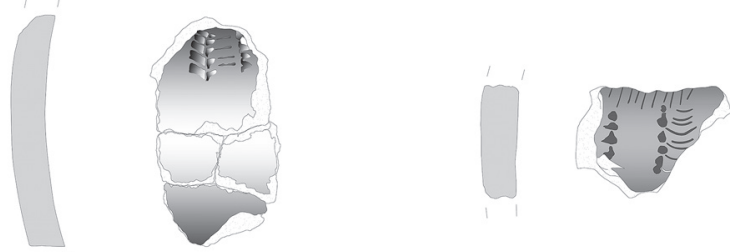

C3-16a-1

C3-16a-2
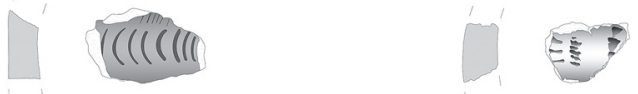

C3-16a-3

C3-16a-4

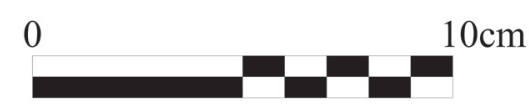

Fig. 8. Muestra representativa en términos morfométricos y decorativos del conjunto cerámico hallado en la primera fase estratigráfica (unidades 16b y 16a). / Representative morphometric and decorative sample of the pottery assemblage recovered in the first stratigraphic phase (Units 16b and 16a). das con diferentes instrumentos y gestos: algunos con una punta simple (Fig. 8: 1, 2 y 4; Fig. 9: 16a-2), otros con el borde de una concha lisa (Fig. 8: 2 y 3; Fig. 9: 16a-2 y 16a-3) y uno con una finísima concha dentada (Fig. 8: 4). Aunque estos fragmentos decorados son de pequeño tamaño, se observa cierta diversidad en los motivos decorados. Algunos de éstos sugieren la posibilidad de que estos fragmentos puedan relacionarse con la denominada ceramica impressa de procedencia mediterránea (ver más adelante).

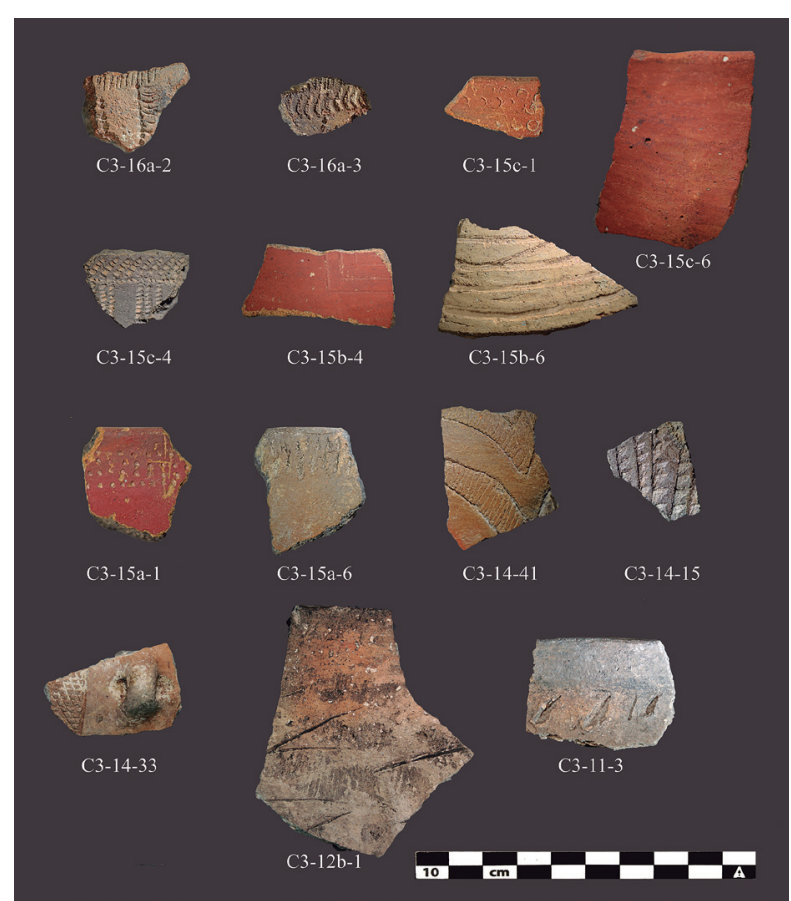

Fig. 9. Fotografías de una selección de piezas cerámicas decoradas correspondientes a toda la secuencia neolítica. Las siglas indican, separados por guiones, el nombre del corte, de la unidad estratigráfica y de la pieza. / Photographs of a selection of decorated pottery fragments from the entire Neolithic sequence. Their identification codes correspond to the excavation area, the stratigraphic unit and the pottery record number, separated by hyphens. 
Las unidades $15 c, 15 b$ y $15 a$, correspondientes a la segunda fase estratigráfica anotada atrás, tienen menos potencia que los estratos inferiores, pero sin embargo cuentan con gran cantidad cerámica y exhiben los porcentajes más elevados de decoradas, así como también cierta diversidad técnica decorativa (Figs. 7A y 7B). Las técnicas más representadas son el englobe a la almagra (Fig. 9: 15c-6, 15b-4 y 15a-1), la incisión (Fig. 9: 15b-4; Fig. 10: 15c-19 y 15c-9; Fig. 11: 15a-2, 15a-21, 15a-5 y 15b-7) y la impresión (Fig. 9: 15c-1, 15c-4 y 15a-1), la aplicación plástica (Fig. 10: 15c-3, 15c-12, 15c-8 y 15c-2; Fig. 11: 15a-14) y finalmente las acanalada (Fig. 9: 15b-6) y excisa (Fig. 9: C3-14-15). Las almagras suelen combinarse con otras técnicas, particularmente con la incisión (Fig. 9: 15b-4; Fig. 11: 15a-2) y con la impresión (Fig. 9: 15a-1). Éstas dos úl- timas también aparecen asociadas (Fig. 10: 15c-21; Fig. 11: 15a-25). Las matrices de las impresiones son diversas, algunas elaboradas con punta simple (Fig. 9: 16a-2 y 15a-1), otras con instrumentos de diversas puntas, como peines o gradinas (Fig. 9: 15c-4), y uno con un elemento tubular (Fig. 9: 15c-1). Los motivos son diversos, pero suelen constituir composiciones de cierta naturaleza geométrica, como líneas o trazos paralelos conformando bandas y más escasamente zigzags o metopas (Figs. 10 y 11). Se trata de fragmentos pertenecientes a recipientes hemisféricos o de tendencia esférica, con predominio de bordes rectos, seguidos de bordes levemente inclinados al interior y presencia mínima de bordes exvasados, cuyos diámetros conocidos se encuentran comprendidos entre un rango de 10 a $30 \mathrm{~cm}$.
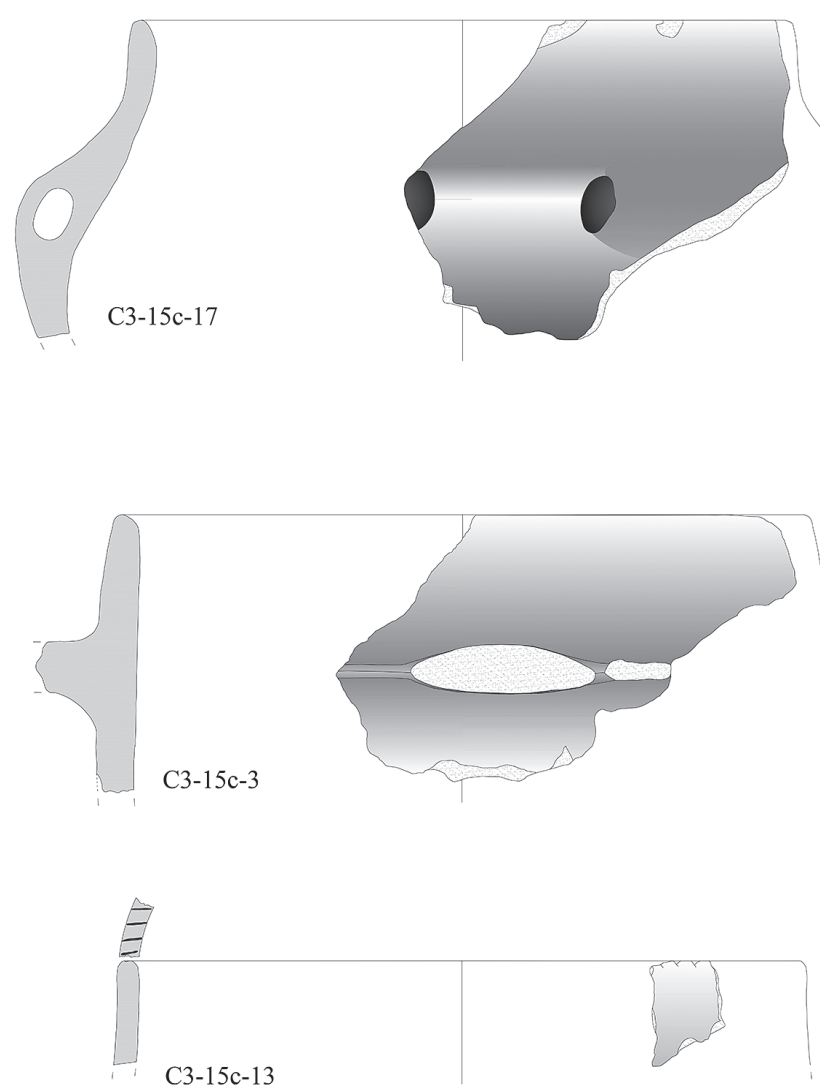

C3-15c-13

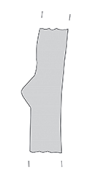

C3-15c-12

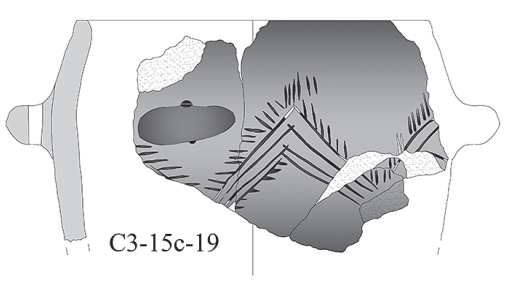

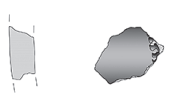

C3-15c-20

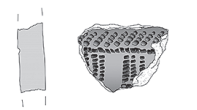

C3-15c-4

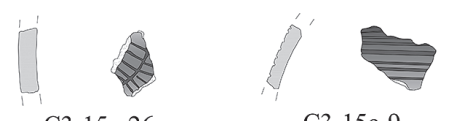

C3-15c-26

C3-15c-9
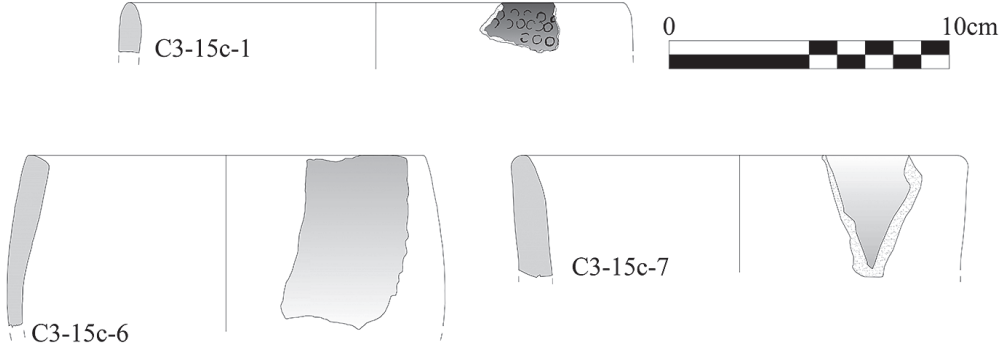

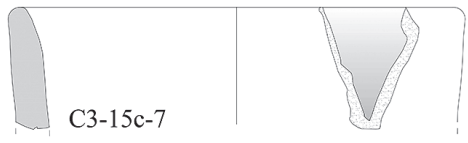

C3-15c-7
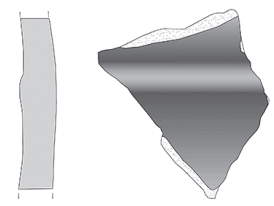

C3-15c-2
Fig. 10. Muestra representativa del conjunto cerámico hallado en la unidad $15 \mathrm{c}$, de la segunda fase estratigráfica. / Representative sample of the pottery assemblage recovered in Unit $15 \mathrm{c}$ of the second stratigraphic phase. 


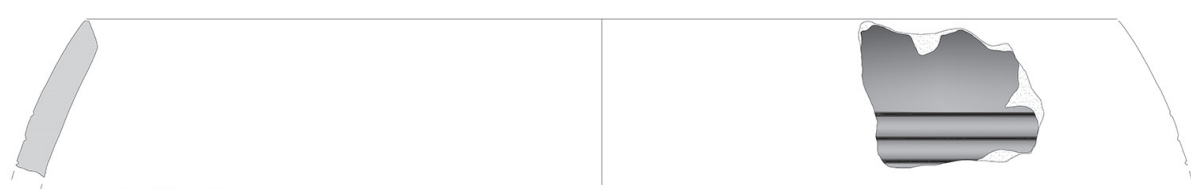

C003-15a-2
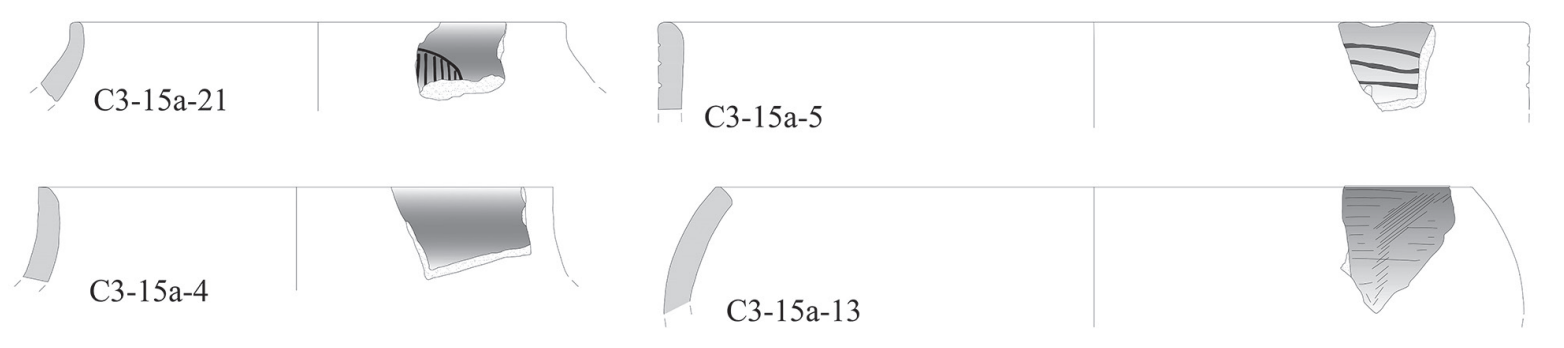

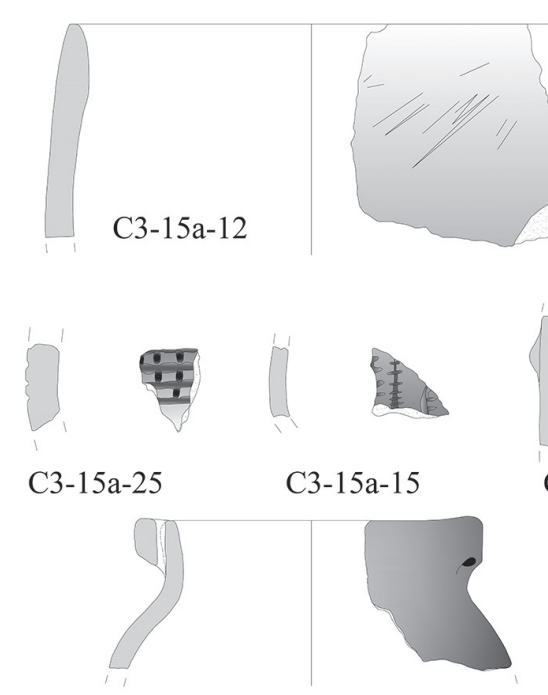

C3-15b-1
C3-15a-14

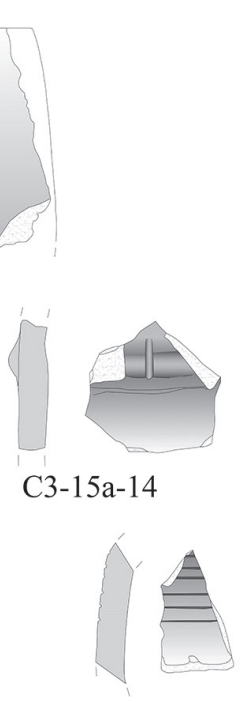

C3-15b-7
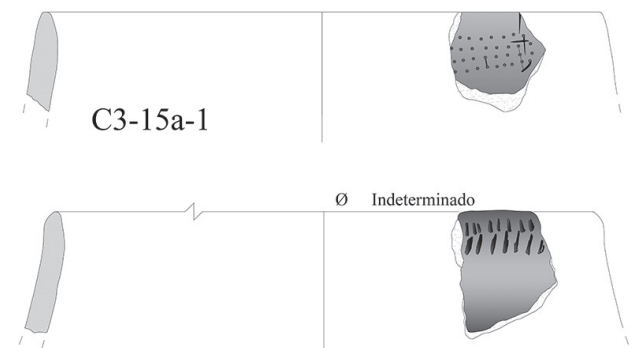

C3-15a-6
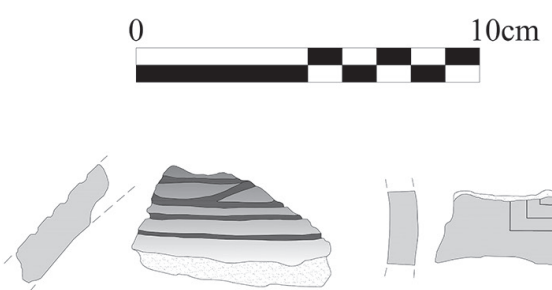

C3-15b-6

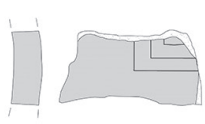

C3-15b-4

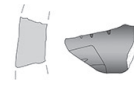

C3-15b-8

Fig. 11. Muestra representativa de las cerámicas hallada en las unidades 15b y 15a, de la segunda fase estratigráfica. / Representative sample of the pottery assemblage recovered in Units $15 \mathrm{~b}$ and $15 \mathrm{a}$ of the second stratigraphic phase.

La tercera fase estratigráfica sugerida arriba engloba una cierta diversidad de estratos $(14,13,12 \mathrm{~b}, 12 \mathrm{a}$, Hogar 1 y 11) que presentan incluso más diferencias internas en cuanto al material cerámico documentado. UE 14 es, de entre aquéllos, el estrato de mayor grosor. Aunque cuenta con alta frecuencia de fragmentos decorados, el porcentaje de éstos respecto al total cerámico de esta unidad no es de los más altos. Sin embargo, es el estrato con mayor diversidad decorativa (Fig. 7). Aparecen, de más a menos, cerámicas incisas (Fig. 9: 14-33; Fig. 12: 4, 42, 35), impresas (Fig. 12: 66), grabadas (Fig. 9: 14-41; Fig. 12: 29-30, 38, 28, 43, 27, 2, 39, 13), excisas -junto con incisiones- (Fig. 9: 14-15; Fig. 12: 17) y finalmente, muy escasas, con aplicación plástica y engobe de almagra. En el resto de estratos de esta fase también predominan las incisas (Fig. 13: 11-11, 12a-10 y 13-3), seguidas de las impresas (Fig. 9: 11-3) -así como ambas conjuntamente (Fig. 13: 13-4)-, y apenas representadas están las técnicas almagra y peinada. En la unidad 12b se encuentra el único fragmento con decoración peinada, que también cuenta con incisiones formando aspas (Fig. 9: 12b-1). UE 14 es el único nivel -no sólo de esta fase sino de toda la secuencia- en donde aparecen cerámicas grabadas. Éste género cerámico destaca entre otros factores por la diversidad y singularidad de sus motivos y composiciones. Éstas suelen estructurarse mediante un concepto de componente más curvilínea que la del geometrismo predominante en otros géneros cerámicos. A juzgar por la escasa información morfométrica disponible (Fig. 12), predominan los recipientes semiesféricos y de tendencia esférica cuyos diámetros en el borde rondan los 20 $\mathrm{cm}$. El estrato 13 presenta principalmente fragmentos de paredes o bordes rectos, cuyos diámetros rondan los $20 \mathrm{~cm}$, mientras que la unidad 12a exhibe formas cerradas, con labios levemente indicados al exterior (Fig. 13). Mientras tanto, en el estrato 11 predominan los recipientes abiertos, de entre 10 y $23 \mathrm{~cm}$ de diámetro en el borde, y existe un ejemplar que probablemente se desvíe un poco del resto de conjunto tipológico (Fig. 13: 11-7) y pueda ser algo más reciente -tal vez procedente de la interfaz inferior del estrato calcolítico 7/10. 


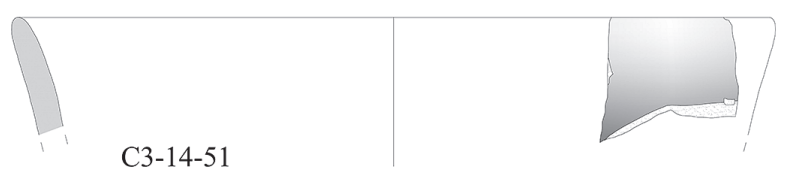

C3-14-66
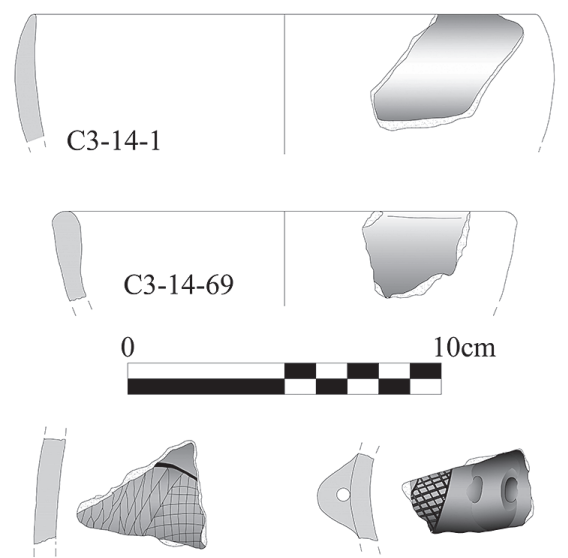

C3-14-39

C3-14-33

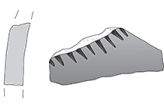

C3-14-35

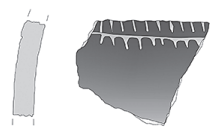

C3-14-42

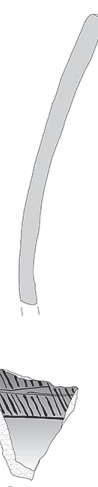

C3-14-38

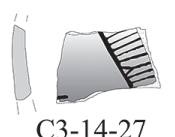

C3-14-27

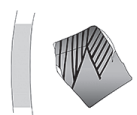

C3-14-13

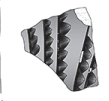

C3-14-15 C3-14-29
C3-14-30

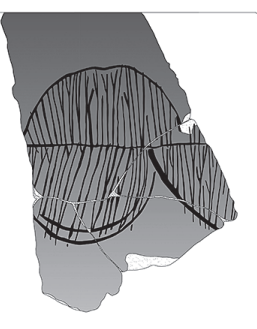

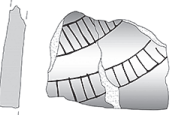

C3-14-28

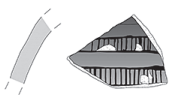

C3-14-43
C3-11-3

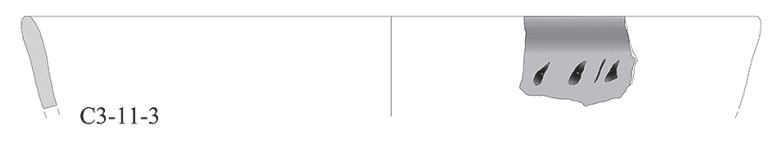

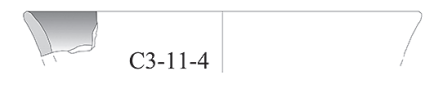

C3-14-2

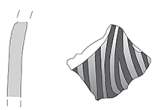

C3-14-4

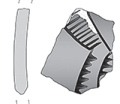

C3-14-36

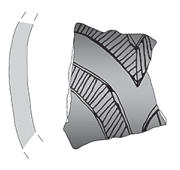

C3-14-41

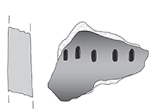

C3-14-18

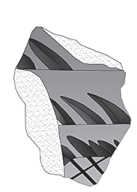

C3-14-17
Fig. 12. Muestra representativa del conjunto cerámico hallado en la unidad estratigráfica 14 , de la tercera fase estratigráfica. / Representative sample of the pottery assemblage recovered in Unit 14 of the third stratigraphic phase.

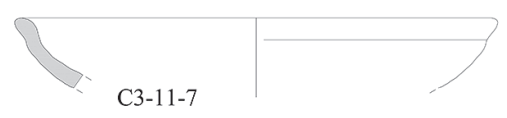

C3-11-7

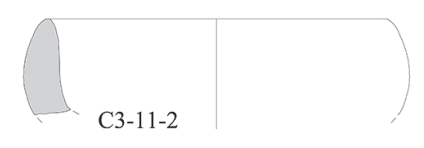

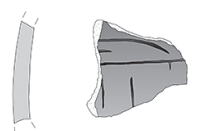

C3-11-11
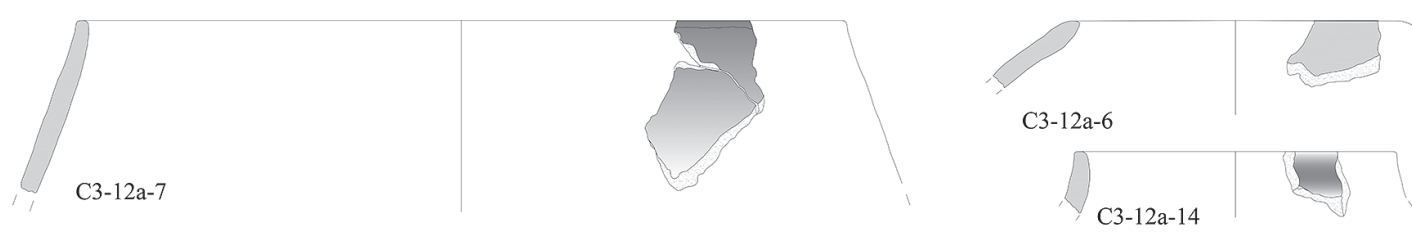

C3-12a-14

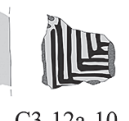

C3-12a-10
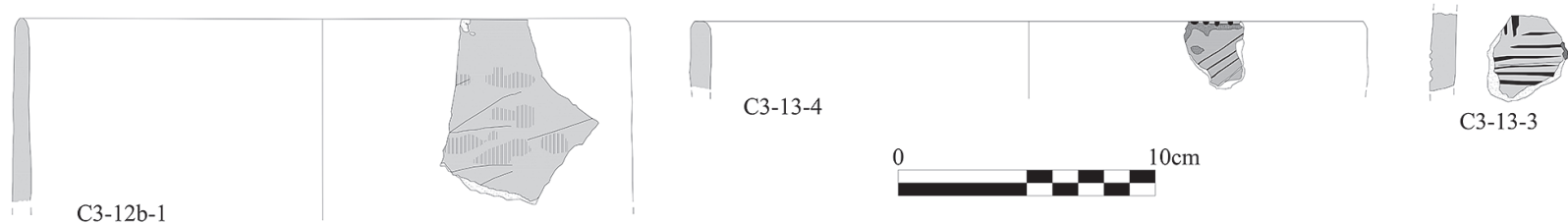

C3-13-4

C3-13-3

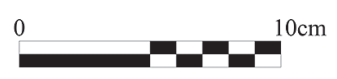

Fig. 13. Muestra representativa de las cerámicas halladas en las unidades estratigráficas 13, 12a, $12 \mathrm{~b}$ y 11 , de la tercera fase estratigráfica. / Representative sample of the pottery assemblage recovered in Units $13,12 a, 12 b$ and 11 of the third stratigraphic phase. 


\subsection{Conjunto lítico}

La industria lítica de los estratos neolíticos de $\mathrm{C003}$ asciende a un total de 153 artefactos, de los cuales 144 son tallados y sólo 9 pulimentados. La presencia de industria lítica tallada es constante, aunque muy variable, a lo largo de secuencia neolítica (Tabla III). La máxima concentración de elementos se constata en los niveles 14, 15a y 15c. Según la secuencia de fases estratigráficas propuestas, la distribución cuantitativa es mayor para la segunda y tercera. Estas cifras marcan un comportamiento diferencial significativo entre los diferentes tramos de la estratigrafía. La lítica pulimentada se halla representada en todas las fases, aunque con discontinuidades en algunos estratos.

De la primera fase estratigráfica sólo proceden 3 elementos de industria tallada y 1 pulimentado. La unidad basal 16b contiene sólo 1 microlasca, mientras que 16a cuenta con 1 gran lasca ancha retocada (Fig. 14: 1), 1 microlaminita muy estrecha que conserva evidencias de uso y, finalmente, una moleta. De la segunda fase estratigráfica (unidades 15c, 15b y 15a), de menor potencia que la anterior, procede sin embargo un porcentaje elevado de lítica tallada (Tabla III, Fig. 14: 2-8) junto a 3 elementos pulimentados. Respecto a su morfometría, de los 68 productos tallados, $30(44,1 \%)$ corresponden al conjunto laminar y $12(17,6 \%)$ al de lascado. El resto está compuesto por 4 núcleos o restos de núcleo $(5,8 \%)$ informes o sobre lasca, 21 restos de talla informes $(30,8 \%)$ y 1 chunk $(1,4 \%)$.

La industria lítica tallada de estas dos fases estratigráficas inferiores (unidades 16b a 15a) está caracterizada por diversos factores. La fragmentación de los soportes afecta a la mitad de las extracciones, con mayor incidencia en las láminas, laminitas y lascas laminares que en las lascas. Los talones conservados, 25 en total, son principalmente lisos (10), facetados (8) diedros (4), puntiformes (2), con 1 caso fracturado. El formato de la industria puede ser calificado como microlítico $(83,8 \%)$ y preponderadamente microlaminar. La materia prima soporte es sílex o cherts de diferentes colores y cali-

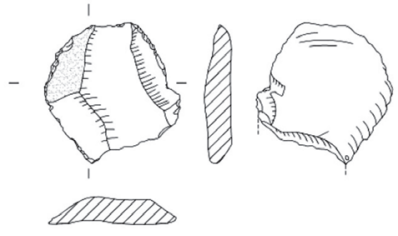

1
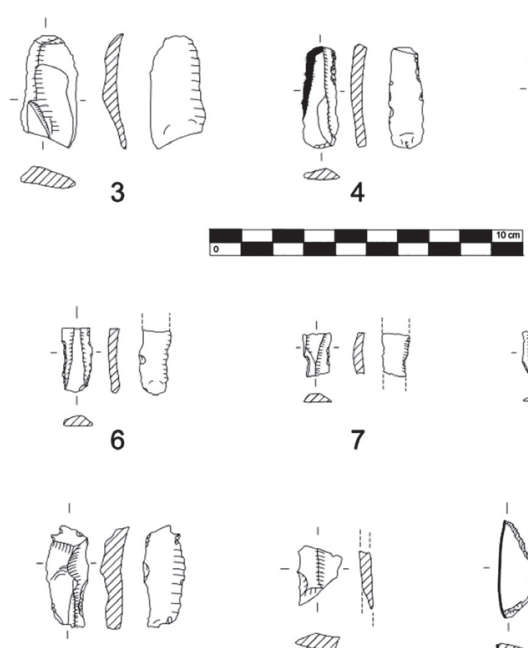

9

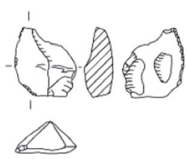

12
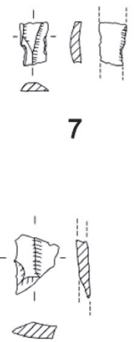

10

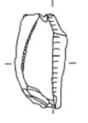

exs

13

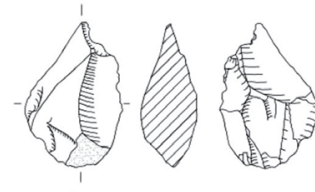

2

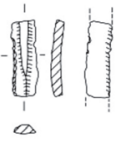

5
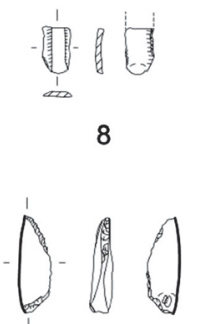

Q20

11

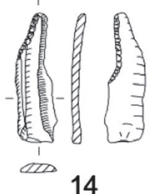

Fig. 14. Selección del conjunto lítico tallado documentado en C003: 1) Lasca de la primera fase estratigráfica); 2 y 3) Perforador y raspador de la segunda fase estratigráfica; 4-8) Láminas y escotadura (8) de la segunda fase estratigráfica; 9-13) Lascas y láminas de la tercera fase estratigráfica; y 14) Perforador de la tercera fase estratigráfica. El córtex de las piezas se representa por trama de puntos, y el sombreado de color negro refleja restos de contacto directo con fuego. / Selection of the knapped stone assemblage documented in C003: 1) Flake, first stratigraphic phase; 2 and 3) Perforator and scraper, second stratigraphic phase; 4-8); Blades and notched blade (8), second stratigraphic phase; 9-13) Flakes and blades, third stratigraphic phase; and 14) Perforator, third stratigraphic phase. Cortex is indicated by the dotted areas, and the blackened areas show direct contact with fire.

\begin{tabular}{|c|c|c|c|c|c|}
\hline $\begin{array}{l}\text { Nombre } \\
\text { estratos }\end{array}$ & $\begin{array}{c}n^{\circ} \text { lítica tallada } \\
\text { (ues) }\end{array}$ & $\begin{array}{c}\mathrm{n}^{\circ} \text { lítica tallada } \\
\text { (fases) }\end{array}$ & $\begin{array}{c}\text { \% lítica tallada } \\
\text { (fases) }\end{array}$ & $\mathrm{n}^{\circ}$ lítica pulimentada (ues) & $\mathrm{n}^{\circ}$ lítica pulimentada (fases) \\
\hline UE 11 & 19 & \multirow{5}{*}{73} & \multirow{5}{*}{$50,7 \%$} & 0 & \multirow{5}{*}{5} \\
\hline UE 12A & 5 & & & 2 & \\
\hline UE 12B & 2 & & & 0 & \\
\hline UE 13 & 1 & & & 0 & \\
\hline UE 14 & 46 & & & 3 & \\
\hline UE 15A & 34 & \multirow{3}{*}{68} & \multirow{3}{*}{$47,2 \%$} & 1 & \multirow{3}{*}{3} \\
\hline UE 15B & 2 & & & 0 & \\
\hline UE 15C & 32 & & & 2 & \\
\hline UE 16A & 2 & \multirow{2}{*}{3} & \multirow{2}{*}{$2,1 \%$} & 1 & \multirow{2}{*}{1} \\
\hline UE 16B & 1 & & & 0 & \\
\hline TOTAL & 144 & 144 & $100 \%$ & 9 & 9 \\
\hline
\end{tabular}

Tabla 3: Conjunto cerámico procedente de los niveles neoliticos del corte C003. / Lithic assemblages from the Neolithic levels of area C003. 
dades en todos los casos, a excepción de un pequeño núcleo agotado de cristal de roca. En al menos el 14\% del material es perceptible claramente el calentamiento del núcleo con carácter previo a la talla. 28 piezas $(39,4 \%)$ han sido conformadas tipológicamente en útiles mediante retoques o bien presentan claros estigmas, huellas o retoques de uso. Se cuenta con 3 raspadores (Fig. 14: 3), 2 elementos de hoz con pátina de siega, 2 troncaduras, 2 buriles diedros, 2 fragmentos de hojitas de dorso, al menos una de ellas apuntada y con estigmas de impacto, 1 pequeña raedera o raclette sobre lasca, 1 escotadura (Fig. 14: 7), 1 perforador atípico o bec (Fig. 14: 2), 1 denticulado, 5 láminas retocadas (Fig. 14: 4-6 y 8) y 8 piezas exclusivamente con retoque de uso.

La tercera fase estratigráfica cuenta con un conjunto de industria lítica tallada de 73 productos y con 5 elementos líticos pulimentados (Tabla III, Fig. 14: 9-14). De las que conforman esta fase, son las unidades extremas 14 y 11 las que con creces cuentan con más industria tallada (46 y 19 productos, respectivamente). En términos morfométricos, hay 2 núcleos, uno informe y el otro agotado sobre lasca (2,7\%); 23 soportes adjudicables al conjunto de lascado (31,5\%), entre ellos una semitableta de acondicionamiento, y un porcentaje similar del laminar $(28,7 \%)$ correspondiente a 21 artefactos. Completan el elenco de productos de talla 25 pequeños restos informes (34,5\%), 1 semiarista y 1 chunk ( $1,3 \%$ cada uno).

La industria lítica tallada de esta fase tiene las siguientes características. Hay 14 lascas y 10 extracciones laminares completas. Los 34 talones presentes como resultado de las fracturas son mayoritariamente lisos (20), diedros (4), puntiformes (4), corticales (2), facetados (2) y fracturados (2). Los soportes suelen contar con morfometrías de pequeño formato y tendencia microlítica $(68,5 \%)$, siempre elaborados en materia prima silícea, y el 5,5\% presenta brillo térmico de precalentamiento de los núcleos. Los útiles retocados propiamente dichos son relativamente más escasos en esta fase, si bien están más significativamente representadas las laminitas, lascas laminares y lascas con huellas de uso y embotamientos ( $26 \%$ en conjunto frente al material no retocado). El primer grupo está compuesto, en términos tipológicos, por 1 elemento de hoz,
1 perforador en extremo de lámina (Fig. 14: 14), 1 troncadura, 1 escotadura, 2 denticulados, 3 dorsos -uno curvo sobre laminita (Fig. 14: 11), una hoja de dorso y un dorso doble sobre lasca-, 1 troncadura cóncava/ bec y 3 lascas retocadas (Fig. 14: 12), mientras que el segundo consta de hasta 5 soportes laminares con retoques de uso (Fig. 14: 9 y 10) y 1 lasca con embotamiento distal. En total, 8 piezas presentan retoques de uso (Fig. 14: 13), 5 diferentes niveles de embotamiento y 1 pátina vegetal.

\subsection{Fechas radiocarbónicas}

Se han llevado a cabo análisis de radiocarbono sobre cuatro muestras procedentes de cuatro estratos diferentes. Se ha fechado el estrato basal de cada una de las fases estratigráficas, es decir UE 16b, 15c y 14, además del estrato superior de la secuencia neolítica -UE 11. Adviértase que los estratos basales de la segunda y tercera fase-UUEE 15 c y 14-, además, se corresponden con los respectivos momentos de aparición de las cerámicas con engobe de almagra, en primer lugar, y las cerámicas grabadas, en segundo lugar.

De estos cuatro estratos se han seleccionado muestras óseas de vida corta en el mejor estado de conservación posible, que resultan ser todas premolares o molares de caprinos (Fig. 15). La identificación taxonómica entre especies dentro de esta subfamilia no está exenta de problemas. Siguiendo el estudio de Zeder y Pilaar (2010) para determinar ovejas y cabras a partir de las mandíbulas, la muestra de UE 11 corresponde a Ovis aries. Las tres muestras restantes parecen también de esta especie, pero pertenecen al maxilar y por tanto es imposible garantizar esta identificación según dicho método. No obstante, la muestra de UE 14 está asociada en el mismo estrato a otras piezas dentales de mandíbula y a una falange III que, según diversos métodos (Boessneck, 1980; Zeder y Lapham, 2010; Zeder y Pilaar, 2010), podrían clasificarse como Ovis aries. Algo similar ocurre con la muestra de UE 15c. En el conjunto de estratos de esta fase estratigráfica se han determinado dos posibles ovejas a partir de piezas dentales, una falange I, una falange II, un fragmento distal de húmero y un fragmento distal de tibia (Boessneck, 1980; Zeder y Lapham, 2010; Zeder

\begin{tabular}{|c|c|c|c|c|c|c|c|c|c|c|c|}
\hline Código & ID & UE & Muestra & $\%$ Rend Extrac. & $\% \mathrm{C}$ & $\% \mathrm{~N}$ & $\mathrm{C}: \mathrm{N}$ & Fecha BP & $\delta 13 C(\% \circ)$ & Cal BC - $1 \sigma$ & Cal BC- $2 \sigma$ \\
\hline CNA4239 & DH16-1 & 11 & $\begin{array}{l}\text { Premolar } 4 \\
\text { - Caprinae } \\
\end{array}$ & 3.34 & 37.21 & 13.7 & 3.2 & $5308 \pm 33$ & $-19.48 \pm 1.50$ & $4135-4056$ & $4241-4042$ \\
\hline CNA4240 & $\mathrm{DH} 16-2$ & 14 & $\begin{array}{l}\text { Molar } 1 \text { - } \\
\text { Caprinae }\end{array}$ & 2.49 & 35 & 13.8 & 3 & $5807 \pm 34$ & $-21.99 \pm 1.50$ & $4713-4649$ & $4728-4549$ \\
\hline CNA4241 & DH16-3 & $15 \mathrm{C}$ & $\begin{array}{l}\text { Molar } 1 \text { - } \\
\text { Caprinae }\end{array}$ & 1.36 & 35.1 & 13.1 & 3.1 & $6609 \pm 35$ & $-20.68 \pm 1.50$ & $5566-5516$ & $5616-5490$ \\
\hline CNA4242 & DH16-4 & $16 \mathrm{~B}$ & $\begin{array}{c}\text { Premolar - } \\
\text { Caprinae }\end{array}$ & 0.8 & 39 & 14.7 & 3.1 & $6541 \pm 34$ & $-20.75 \pm 1.50$ & $5521-5477$ & $5561-5470$ \\
\hline
\end{tabular}

Tabla 4: Fechas radiocarbónicas efectuadas sobre las muestras de la campaña arqueológica de 2016. Calibración efectuada con Calib 7.0 (Reimer et al., 2013). / Radiocarbon dates from the samples recovered during the 2016 field work. Calibration performed with Calib 7.0 (Reimer et al., 2013 ). 


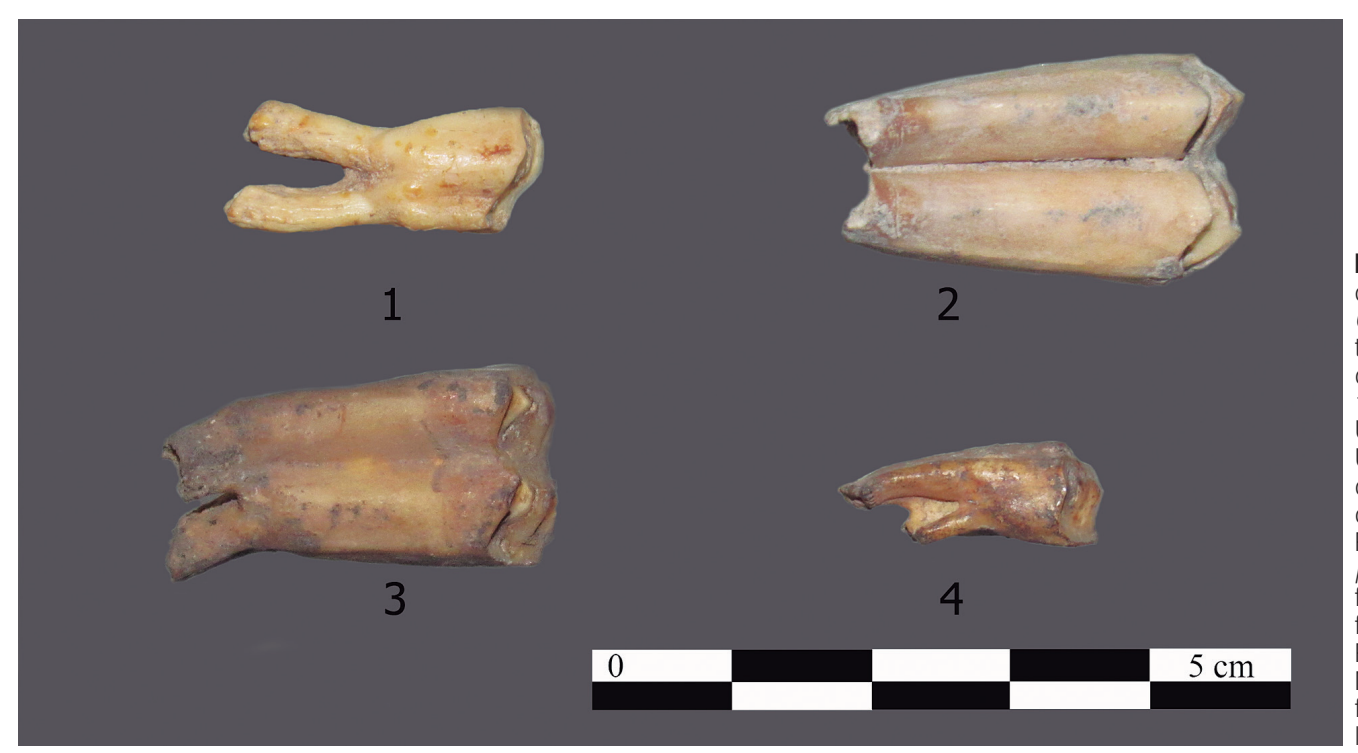

Fig. 15. Fotografías de las cuatro piezas dentales de Caprinae usadas para la extracción de muestras para dataciones radiocarbónicas: 1) Premolar 4 procedente de UE 11 ; 2) Molar 1 hallado en UE 14; 3) Molar 1 encontrado en UE 15c; y 4) Premolar descubierto en UE 16b. Photographs of the four $\mathrm{Ca}$ prinae dental elements used for the extraction of samples for radiocarbon dating: 1) Premolar 4 from Unit 11; 2) Molar 1 from Unit 14; Molar 1 from Unit 15c; and 4) Premolar from Unit $16 \mathrm{~b}$.

y Pilaar, 2010). Estas identificaciones provisionales, así como la de la muestra procedente de UE 16b, podrán contrastarse a partir de los análisis genéticos que actualmente se llevan a cabo. Las muestras se han analizado en el Centro Nacional de Aceleradores, Sevilla. Los resultados se reflejan en la Tabla IV. Las fechas se refieren a la edad de muerte de los especímenes analizados, y se asumen como indicadores cronológicos de los estratos que los contienen.

Las fechas correspondientes a los estratos inferiores se solapan indicando la proximidad cronológica de las dos primeras fases estratigráficas. La primera, datada a partir de la unidad 16b, arroja el intervalo 55615470 cal BC a dos sigmas, mientras que la segunda, muestreada en UE 15c, indica el intervalo 5616-5490 cal BC a dos sigmas. Por tanto, puede mantenerse un Neolítico antiguo en este sitio arqueológico con fechas algo anteriores a la mitad del VI milenio cal BC.

El inicio de la tercera fase estratigráfica queda materializado en UE 14 con la fecha $4728-4549$ cal BC a dos sigmas. Este nivel supone un punto de inflexión en la secuencia estratigráfica según la cantidad de material arqueológico documentado y también según la aparición de algunos ítems particulares, como las cerámicas grabadas. Por último, UE 11 supone la fecha neolítica más reciente del sondeo, arrojando la cifra 4241-4042 cal BC a dos sigmas. Por tanto, la tercera fase estratigráfica quedaría enmarcada por estas dos últimas fechas, ubicándose pues en el $\mathrm{V}$ milenio cal BC.

El lapso temporal entre ambas fechas radiocarbónicas, de aproximadamente 500 años, no puede saberse actualmente si se correspondería con las deposiciones de los estratos intermedios (13, 12b, 12a y Hogar 1), aún no datados, y entre los que existen algunas evidencias directas de ocupación -unidades 12b y Hogar 1-; o si, por el contrario, se relacionaría con el presunto hiato poblacional propuesto generalmente para el Neolítico medio (ver más adelante).

\section{DISCUSIÓN}

Los datos sobre la secuencia neolítica de Cueva de la Dehesilla obtenidos en 2016 pueden compararse tentativamente con aquellos publicados de las primeras excavaciones de 1977 y 1981. No obstante, dicha comparación ha de llevarse a cabo con cautela. Primero por las reducidas dimensiones espaciales del corte C003; segundo, porque éste se ubica aproximadamente a $10 \mathrm{~m}$ y $20 \mathrm{~m}$ de los cortes A (de 1977) y B (de 1981), respectivamente, distancias más que suficientes para que los pormenores estratigráficos puedan variar sensiblemente entre aquél y éstos.

Las dos fases más antiguas de la secuencia, compuestas por las unidades 16b y $16 \mathrm{a}$-la primera-, y 15c, 15b y $15 a$-la segunda-, comparten algunas características sedimentológicas y también cerámicas. En cuanto a lo primero, son todos estratos de color marrón, aunque de diferentes tonalidades; todos cuentan con una alta presencia de bloques calizos, aunque son menores y más pequeños en 15a. En cuanto a las cerámicas, excepto el inferior, cuentan con los porcentajes más altos de fragmentos decorados. Los dos análisis radiocarbónicos de $16 b$ y 15c coinciden en fechas de la mitad del VI milenio cal BC. Tanto el conjunto cerámico como las dos fechas indican un periodo de Neolítico antiguo.

No obstante, existen algunas diferencias entre ambas fases estratigráficas, no sólo en cuanto a características sedimentológicas sino también respecto a la cultura material. La primera se caracteriza por la textura arenosa que comparten los estratos 16b y 16a, mientras que la segunda por la naturaleza arcillosa de los estratos $15 \mathrm{c}, 15 \mathrm{~b}$ y $15 \mathrm{a}$. Sólo la primera fase (particularmen- 
te el estrato 16a) cuenta con fragmentos posiblemente de ceramica impressa, y no con cerámica a la almagra, que es de hecho la decoración más representada en los estratos de la segunda fase. Existe igualmente cierta diferencia entre las cantidades de industria lítica tallada, siendo ésta más numerosa en la segunda fase. A la espera de los resultados de las nuevas campañas de excavación, pueden asumirse momentáneamente tales diferencias y proponer dos periodos de Neolítico antiguo, A y B.

En cualquier caso, la presencia de ceramica impressa abre el debate a una serie de puntos relevantes. A nivel local y regional, plantea la relación y cronología relativa entre aquélla, la cerámica cardial y la cerámica a la almagra. Según los escasos datos obtenidos en este sondeo, la almagra se superpone a la impressa, lo que pondría en entredicho el paradigma de que las cerámicas a la almagra indiquen necesariamente el primer neolítico del sur peninsular, especialmente del suroeste (Pellicer y Acosta, 1990) (pero véase Gutiérrez-López et al., 1996), aunque efectivamente sean aquí el género decorado más común durante el Neolítico antiguo. El único fragmento que presenta, entre otras técnicas decorativas, impresión mediante concha dentada -aunque no se trate de un espécimen típicamente cardial- se ubica también en el estrato subyacente. Otro punto de debate que se dinamiza, en este caso a nivel peninsular, es el área de distribución de la ceramica impressa, que se conoce en el Mediterráneo occidental, desde los mares Jónico y Adriático (e.g. Berger et al., 2014), al litoral ligur francés (Guilaine y Manen, 2002; Guilaine et al., 2007; Binder et al., 2017), hasta la península ibérica. Sin embargo, aquí se encuentran escasamente en el litoral este (cf. Bernabeu et al., 2009; 2011), sin que hasta hoy se hayan documentado al sur de la región valenciana (cf. García Borja et al., 2014).

Las excavaciones efectuadas en los años 1977 y 1981 permitieron proponer igualmente dos periodos de Neolítico antiguo, A y B, que se correspondían respectivamente con los estratos VI y $\mathrm{V}$ documentados en los dos cortes (Acosta y Pellicer, 1990: 24 y ss.). Ambos estratos eran de textura arcillosa y color marrón, aunque con presencia esporádica de bloques -los mayores tamaños y proporciones de bloques se observaron en la base sobre la que se depositó el estrato basal VI. Los conjuntos cerámicos de los estratos VI y V, aunque con sutiles diferencias porcentuales entre sí (cf. Acosta y Pellicer, 1990: 69 y ss.), coincidían ambos, no obstante, en la mayoría de aspectos considerados: predominio de cerámicas toscas con decoraciones plásticas, de formas de hombros entrantes cóncavos, de asas de cinta y, más próximos a las unidades de nuestro análisis, de cerámicas a la almagra, seguidas de aplicaciones plásticas, incisas, acanaladas, impresas y cardialoides (Acosta y Pellicer, 1990: 99-100). En dichas excavaciones la cerámica a la almagra aparecía desde los estratos basales de sendos cortes y no se identificó fragmento alguno de ceramica impressa.
El descubrimiento novedoso de dicho género cerámico -asociado a lo largo del Mediterráneo occidental a las primeras poblaciones neolíticas- y las nuevas fechas radiocarbónicas convergen en indicar una temprana ocupación en Cueva de la Dehesilla relacionada con los albores del proceso de neolitización peninsular. En el entorno próximo a la cueva existen sitios con ocupación de Neolítico antiguo (Fig. 16 y Tabla V). Entre la Sierra de Grazalema y el piedemonte Subbético occidental, se conocen Cueva del Parralejo, en San José del Valle (Pellicer y Acosta, 1982: 55; Acosta, 1995: 65), Cueva del Picado, en Jerez de la Frontera (Mora-Figueroa, 1970), la Sima S-6 de Veredilla, en Benaocaz (Guerrero-Misa, 1992), así como en la campiña los sitios al aire libre Cabezo de Hortales, en Prado del Rey, La Escalera, en Arcos de la Frontera, y por último Esperilla y Los Frailes, ambos en Espera (Gutiérrez-López et al., 1996; 2000). Desafortunadamente, sólo la Cueva del Parralejo ha sido objeto de excavaciones arqueológicas, practicadas en el año 1977 pero sin apenas publicar, y no cuenta con dataciones radiocarbónicas. Hacia el oeste, en la línea costera de la margen izquierda de la paleodesembocadura del Guadalquivir, se conocen algunos sitios al aire libre. El Cabezo, en el casco actual de Lebrija, se excavó en 1986 (Caro et al., 1987; Escacena y García-Rivero, en prensa), pero no se llevaron a cabo fechas radiocarbónicas. Existen noticias de hallazgos superficiales, supuestamente del mismo periodo, en Los Pozos (Caro, 1991: 63; Acosta, 1995: 35), así como en Bustos (Lavado, 1990: 126) y Alventus-El Nono (Escacena, 2014: 86-87; Gómez-Peña et al., 2014: 263), estos dos últimos en el término de Trebujena.

De la tercera fase estratigráfica, las unidades 14 , $13,12 a$ y 11 , que constituyen auténticos estratos de tendencia horizontal, comparten diversas características sedimentológicas. Se trata de niveles de textura arcillosa muy compactada, de deposición lenta y cuyos colores -al contrario de las tonalidades marrones de las dos fases anteriores- comparten gamas grises, si bien algunos muestran matices amarillentos (UE 13) o anaranjados (UE 11). Tienen además el rasgo común de poseer una gran cantidad de materia orgánica, si bien el superior (UE 11) contiene menos restos de carbón. Los dos análisis radiocarbónicos efectuados a las unidades inferior y superior de esta fase (14 y 11, respectivamente) indican un intervalo cronológico que ocupa los tres últimos tercios del $\mathrm{V}$ milenio cal $\mathrm{BC}$, fechas que con el conjunto de materiales arqueológicos documentados son coherentes con la asignación de un periodo de Neolítico medio para esta fase estratigráfica.

Sin embargo, la cultura material de este conjunto de estratos muestra notables diferencias internas. Los niveles inferior y superior (14 y 11) de esta fase estratigráfica son de los que contienen mayor cantidad cerámica, que decrece notablemente en los estratos intermedios. La proporción de cerámica decorada parece disminuir en los niveles superiores: $13,08 \%$ en UE 14; $17,85 \%$ en UE 13; 9,18\% en UE 12a; y 2,55 en UE 11. 


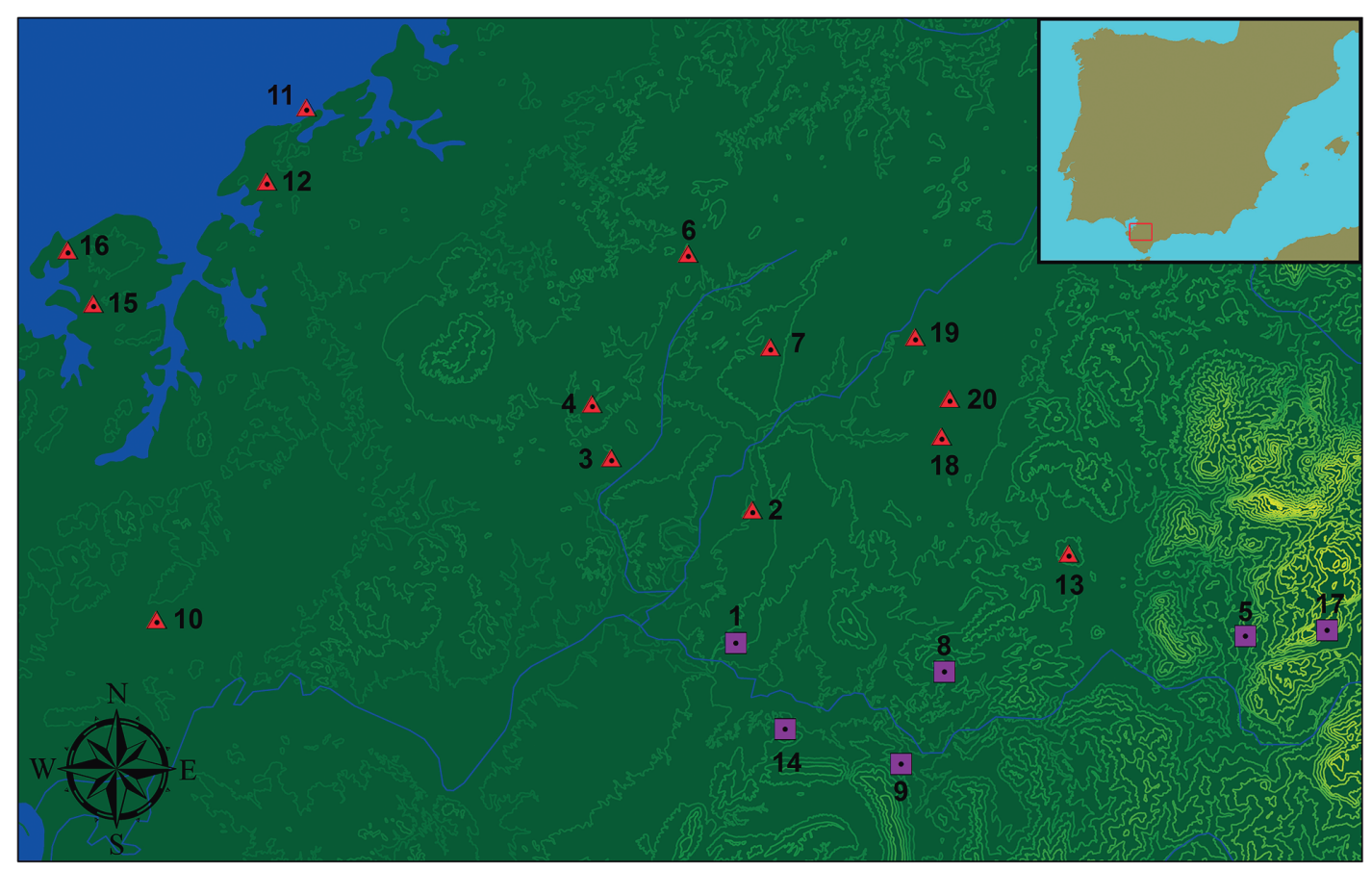

- Yacimiento en cueva

4. Yacimiento al aire libre

\begin{tabular}{|c|c|c|c|}
\hline id. & yacimiento & $\begin{array}{c}\text { Neolítico } \\
\text { Antiguo } \\
\text { vi milenio bc }\end{array}$ & $\begin{array}{c}\text { Neolítico } \\
\text { Medio-final } \\
\text { v/iv milenios bc }\end{array}$ \\
\hline 1 & Cueva del Higueral de la Valleja & \\
\hline 2 & La Escalera & \\
\hline 3 & El Jadramil & \\
\hline 4 & El Yugo & \\
\hline 5 & Sima S-6 de La Veredilla & \\
\hline 6 & Esperilla & \\
\hline 7 & Los Frailes & \\
\hline 8 & Cueva de la Dehesilla & \\
\hline 9 & Cueva del Picado & \\
\hline 10 & Armas de Santiago & \\
\hline 11 & El Cabezo & \\
\hline 12 & Los Pozos & \\
\hline 13 & Cabezo de Hortales & \\
\hline 14 & Cueva del Parralejo & \\
\hline 15 & Bustos & \\
\hline 16 & Alventus-El Nono & \\
\hline 17 & Cueva VR-15 & \\
\hline 18 & El Convento & \\
\hline 19 & Cerro de la Gloria & \\
\hline 20 & Alberite & \\
\hline
\end{tabular}

Tabla 5: Seriación cronológica de las ocupaciones neolíticas en el entorno regional próximo. / Chronological seriation of the Neolithic sites of the surroundings near Dehesilla Cave.
Fig. 16. Sitios arqueológicos del entorno próximo a Cueva de la Dehesilla citados en el texto. Archaeological sites mentioned in the text of the surroundings near Dehesilla Cave.

Los dos estratos inferiores (14 y 13) mantienen ejemplares de algunos géneros decorativos presentes en la fase anterior: ambos tienen residualmente alguna cerámica a la almagra, pero sólo en el más antiguo perviven aplicaciones plásticas y la técnica excisa. UE 14 es también el único estrato de toda la secuencia que contiene cerámica grabada. El resto de estratos de esta tercera fase sólo presentan, en cambio, cerámicas incisas e impresas comunes, a excepción de un fragmento con decoración peinada hallado en la unidad 12b. La información morfológica indica también cierta diferencia del estrato superior, UE 11, respecto del resto, porque en aquél son usuales los recipientes abiertos. En cuanto a la industria lítica tallada, también son los estratos 14 y 11 los que cuentan con conjuntos más sustanciosos. UE 11 presenta algunas diferencias respecto a los estratos anteriores. En dicha unidad no se constata la presencia de precalentamiento de los núcleos, ni en los soportes propiamente dichos, ni en los restos de talla. Igualmente, la anterior tendencia morfométrica microlítica resulta en este caso invertida, con tan sólo 3 pequeños micro-formatos mensurables frente a 4 lascas y 4 láminas.

Por tanto, a la espera de nuevos datos procedentes de las excavaciones futuras del proyecto, es posible sugerir a modo tentativo una periodización interna también para el Neolítico medio. En primer lugar, se puede proponer consistentemente un periodo Neolítico medio A, correspondiente con la unidad 14 (con la fecha radiocarbónica 4728-4549 cal BC) y cuya cantidad de cultura material tanto cerámica como lítica indica una 
ocupación intensa de la cavidad. Este periodo está singularmente caracterizado por las cerámicas grabadas. Este género cerámico, no obstante, apenas se conoce en el sur peninsular, y con composiciones decorativas similares a las Cueva de la Dehesilla sólo lo conocemos actualmente en los sitios cercanos de El Parralejo (San José del Valle) (Pellicer y Acosta, 1982: 55; Acosta, 1995: 65; Amores, 2009) y Esperilla (Espera) (Gutiérrez-López et al., 1996).

En segundo lugar, se puede proponer un segundo periodo, Neolítico medio B, materializado en los estratos $13,12 \mathrm{~b}$ y $12 \mathrm{a}$, y cuya característica principal es el notable descenso cuantitativo de los propios conjuntos materiales. No sabemos si este periodo se podría relacionar con el anteriormente mencionado hiato poblacional del Neolítico medio. En este sentido, en la región andaluza, es de especial relevancia el sitio de Los Castillejos, en Las Peñas de los Gitanos (Montefrío, Granada), por el intenso programa de actividades arqueológicas llevadas a cabo, y donde existe un abandono del sitio entre aproximadamente 4800-4400/4200 cal BC (Molina et al., 2017). De existir un hiato similar en Cueva de la Dehesilla -que aún no sabemos-, éste habría de situarse en cualquier caso un poco más tarde, a partir de la mitad del $\mathrm{V}$ milenio cal BC.

Por último, el estrato 11 es de difícil asignación en la periodización. Recobra los valores cuantitativos materiales, si bien sus características difieren notablemente de las anteriores. Apenas existen cerámicas decoradas, y son recipientes usualmente abiertos. La industria lítica no presenta, por vez primera en la secuencia, brillo térmico, reflejando la pérdida de la técnica del precalentamiento de núcleos. Si bien estos rasgos podrían indicar un periodo de Neolítico final, este estrato ha arrojado la fecha 4241-4042 cal BC. Además, entre el conjunto cerámico aparece un fragmento propio de época calcolítica -tal vez procedente del estrato superior mediante la afectación de la madriguera mencionada atrás. A la espera de los resultados de las próximas intervenciones arqueológicas en el sitio, mantenemos la reserva con este estrato y lo clasificamos provisionalmente como Neolítico medio-final.

La documentación arqueológica llevada a cabo en las campañas de 1977 y 1981 permitió sugerir dos periodos de Neolítico medio, A y B, y uno de Neolítico reciente, correspondientes con los estratos IVB, IVA y III, respectivamente. El denominado IV contaba con una matriz de arcilla parda-grisácea, y en el corte de 1981 se subdividió en dos subestratos, IVB y IVA, porque el primero e inferior contaba con menor cantidad y tamaño de bloques calizos, así como con matices rojizos y abundantes hogares y zonas carbonatadas (Acosta y Pellicer, 1990: 25-26). El estrato III contaba con una matriz arcillosa, parda-amarillenta o parda-rojiza según los cortes. De nuevo, como ocurría en la subdivisión de Neolítico antiguo que efectuaron estos autores, los dos estratos entonces entendidos como Neolítico medio mostraban, no obstante, proporciones similares entre
Sí en cuanto al tratamiento y cuidado de las cerámicas (Acosta y Pellicer, 1990: 73 y ss.), así como en la proporción relativa de géneros cerámicos, predominando la incisión, seguida de la impresión, la aplicación plástica, la almagra, la acanalada y grabada, y, finalmente, la cardialoide (Acosta y Pellicer, 1990: 100 y ss.). El estrato del que denominaron Neolítico reciente se caracterizaba por recipientes cerámicos de borde quebrado, entre otros, y por un claro predominio de cerámicas incisas, seguidas de acanaladas, impresas, almagroides y finalmente plástica (Acosta y Pellicer, 1990: 102), en claro contraste con nuestra unidad 11.

Respecto a estos periodos neolíticos más avanzados, en el entorno próximo a Cueva de la DehesiIla, algunos de los sitios previamente mencionados cuentan también con ocupaciones de Neolítico medio, como por ejemplo la Cueva del Parralejo, así como los sitios al aire libre de Cabezo de Hortales y Esperilla. Hay que mencionar otros emplazamientos que parecen ocuparse por vez primera ya en fechas finales del $\mathrm{V} o$ principios del IV milenio BC. Próximos a la Cueva de la Dehesilla por la parte septentrional, en el municipio de Villamartín, se conocen evidencias superficiales en los sitios al aire libre de El Convento y Cerro de la Gloria (Gutiérrez-López et al., 2000), así como en el megalito de Alberite (Ramos y Giles, 1996). Hacia el este, en plena Sierra de Grazalema, se conocen numerosos sitios, entre los que destacan las cuevas VR-15, en Villaluenga del Rosario, con evidencias interesantes referentes a las dimensiones funeraria y ritual de estas poblaciones (Santiago et al., 1997; Gutiérrez-López et al., 2000), así como la del Fantasma o Sima S-6 de La Veredilla, ubicada en Benaocaz, que contiene, aunque desafortunadamente procedente de superficie, un amplio e interesante conjunto de material arqueológico (Guerrero-Misa, 1992; Gutiérrez-López et al., 2000). Hacia el oeste, en el término de Arcos de la Frontera, se dispone de algunas evidencias a partir de varios sondeos estratigráficos en la Cueva del Higueral, en Sierra de la Valleja (Giles et al., 1996: 40-41), así como en la fase más antigua del extensamente excavado asentamiento de El Jadramil (Lazarich y Richarte, 2003), y también en los datos superficiales de El Yugo (Perdigones, 1987). En esta misma zona de campiña, pero en el término de Jerez de la Frontera, se excavó el sitio de Armas de Santiago, con una fase estratigráfica prehistórica conformada por estructuras tipo silo cuyo material de relleno motivó la datación tentativa de transición entre $V$ y IV milenios BC (Pérez-Rodríguez et al., 2010).

\section{CONCLUSIONES}

Los datos presentados proceden de un sondeo (C003) practicado en un área intermedia del interior de la Cueva de la Dehesilla durante las excavaciones de 2016. Las dimensiones reducidas del sondeo limitan la lectura de los datos expuestos a una perspectiva estratigráfica vertical. Por tanto, la discusión planteada atrás 
es de carácter preliminar, sujeta a los nuevos datos que se vayan extrayendo durante las siguientes campañas del proyecto. La extraordinaria potencia documentada en el sondeo, con $4 \mathrm{~m}$ de secuencia prehistórica, de ellos $3 \mathrm{~m}$ específicamente neolíticos, consolidan la relevancia y el potencial de este sitio arqueológico.

A partir de la secuencia estratigráfica, los materiales -principalmente cerámicos- y las dataciones absolutas sobre elementos de vida corta, podemos proponer una secuencia cronológico-cultural que cuenta con dos fases estratigráficas superpuestas correspondientes al Neolítico antiguo. Las dos dataciones disponibles fueron realizadas sobre piezas dentales de Caprinae seleccionadas de la unidad estratigráfica más antigua UE 16b y del estrato basal de la fase suprayacente (UE 15c), por lo que son indicativas de los inicios de la ocupación de Neolítico antiguo, pero no representativas de todo el lapso temporal de este periodo. Ambas dataciones, muy próximas entre sí, muestran un rango temporal solapado que abarca los siglos que median el VI milenio cal BC (5616-5470 cal BC), coincidiendo con la datación más antigua sobre elemento doméstico disponible hasta la fecha para el neolítico andaluz procedente de la Cueva de Nerja (Beta-131577, 6590 \pm 40 BP, Ovis aries) (Aura et al., 1998).

Sin embargo, a diferencia del yacimiento malagueño, las dos fases estratigráficas de Cueva de la DehesiIla se diferencian tanto por aspectos sedimentológicos como especialmente por su contenido artefactual, lo que sugiere la existencia de dos realidades culturales distintas. La más reciente coincide con Nerja en contener un conjunto de cerámicas incisas, impresas, con aplicaciones plásticas y engobe a la almagra, típico del Neolítico antiguo andaluz; mientras que la más antigua carece de estos dos últimos géneros, y en cambio presenta un pequeño pero significativo conjunto de fragmentos cerámicos que comparten unos mismos rasgos técnicos combinando incisiones e impresiones realizadas mediante diferentes instrumentos, concha lisa y concha dentada, y cuyo estilo se asemeja a determinadas producciones típicas del complejo de la ceramica impressa mediterránea.

Esta plausible filiación conlleva novedosas implicaciones sobre el estudio de las primeras poblaciones neolíticas de la región, así como también sobre su(s) posible(s) procedencia(s). Pero también atañe al estudio general del movimiento e interacción de las primeras poblaciones campesinas y ganaderas próximas a las zonas litorales de la península ibérica en el marco del Mediterráneo occidental. Significativamente, los recientes análisis genómicos de individuos pertenecientes a las poblaciones del Neolítico antiguo andaluz de las Cuevas de Murciélagos de Zuheros (Valdiosera et al., 2018) y de la Cueva del Toro (Fregel et al., 2018) indican que la filiación de los agricultores y ganaderos responsables del Neolítico a la almagra es la misma que la de los neolíticos cardiales de las zonas más septentrionales de la costa mediterránea y del interior de Iberia.
En la estratigrafía obtenida hemos documentado también una secuencia de Neolítico Medio, cuyas fechas radiocarbónicas abarcan los tres últimos cuartos del V milenio cal BC. Su inicio conforma un subperiodo que hemos denominado Neolítico medio A materializado en un estrato (UE14) fechado por radiocarbono en 4728-4549 cal BC. Éste es relevante no sólo por su propia fecha, en un lapso temporal del que se tienen escasos datos de ocupaciones en el sur de Iberia, sino también porque dispone exclusivamente de un amplio conjunto de cerámicas grabadas, que presentan unos atributos técnicos y estilísticos muy marcados, con unos motivos y composiciones decorativas peculiares, que actualmente se conocen sólo en otros dos yacimientos y se constriñen a un entorno local muy focalizado. Ambos factores sugieren la posibilidad de una o varias poblaciones con relativo grado de aislamiento, bien por una tasa baja de transmisión cultural interdémica en este periodo y/o por un escenario de escasa ocupación demográfica sobre el territorio.

Este periodo específico cuenta con escasos paralelos temporales en yacimientos bien datados a partir de elementos domésticos y/o de vida corta, no sabemos pues si relacionándose con el hiato poblacional propuesto recientemente (Molina et al., 2017). Existen algunas escasas dataciones que abarcan el segundo y tercer cuarto del $\mathrm{V}$ milenio cal BC diseminadas por yacimientos en cueva y al aire libre de la costa mediterránea y el sureste andaluz, y recientemente se ha publicado una nueva datación que marca el final de la ocupación neolítica del yacimiento al aire libre del Castillo de Doña Mencía. Ubicado como Cueva de la Dehesilla en el subbético externo, adquiere especial relevancia comparativa al superponerse parcialmente su cronología a la fase de Neolítico Medio A que hemos descrito. Igualmente realizada sobre Caprinae arroja la

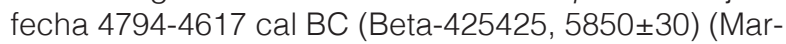
tínez y Vera, 2017: 25), y su interés estriba en que los contextos cerámicos del yacimiento no muestran afinidades técnicas ni estilísticas con las que nos ocupan, sino que mantienen la tradición del Neolítico antiguo ya bien entrado el $\mathrm{V}$ milenio cal BC.

El siguiente y último cambio notable de la secuencia neolítica se materializa en un estrato fechado por radiocarbono a finales del $\mathrm{V}$ milenio (4241-4042 BC cal BC), cuyos conjuntos cerámicos y líticos presentan unas características distintivas respecto a los anteriores. En lo que respecta a la alfarería destaca el cambio que se produce en las formas de las cerámicas, de mayor tendencia abierta, así como la notable reducción numérica de las decoraciones. Muy llamativo es igualmente el abandono de la técnica de precalentamiento de los núcleos para la producción de herramientas líticas, así como la considerable reducción numérica de las morfometrías microlíticas.

En términos generales, esta secuencia cronológica cultural basada en el sondeo C003 converge con el resumen de la propuesta esgrimida por Acosta y 
Pellicer (1990) en las excavaciones de 40 años atrás, reafirmando sobre todo la importancia de este enclave arqueológico en relación con diversos puntos de discusión historiográfica relativos al estudio de las poblaciones neolíticas de la península ibérica. No obstante, también existen ciertos contrastes, sobre todo en cuanto a las posiciones relativas entre algunos géneros y tipos de artefactos, y a sus diferentes evoluciones temporales y porcentuales. Aunque las nuevas excavaciones arqueológicas de Cueva de la Dehesilla han permitido corroborar la relativa antigüedad de sus primeras poblaciones campesinas y ganaderas, desmienten la posibilidad de una filiación autóctona o invención local de la economía productora (Acosta, 1987), y proporcionan nuevos datos relativos a las raíces mediterráneas de los inicios del Neolítico antiguo andaluz.

\section{AGRADECIMIENTOS}

Diversas entidades y programas de la Universidad de Sevilla han financiado la campaña arqueológica del año 2016: Ayudas de Innovación y Mejora Docente 2016, Ref. 1.10 del Plan Propio de Docencia; Ayudas para Acciones Especiales 2016, Ref. IV.7 del V Plan Propio de Investigación; Actividades Culturales 2016, Facultad de Geografía e Historia; Departamento de Prehistoria y Arqueología; y Grupo Tellus: Prehistoria y Arqueología en el Sur de Iberia, HUM-949 Plan Andaluz de Investigación. C. Umbelino cuenta usualmente en sus investigaciones con el apoyo de la FCT (UID/ANT/00283/2013).

Hay un gran conjunto de personas e instituciones que han colaborado o participado en la campaña de 2016. El Ayuntamiento de Algar ha colaborado con la ayuda de medios humanos y materiales en diversas tareas logísticas. El Ayuntamiento de Jerez de la Frontera ha prestado puntualmente su servicio topográfico. La familia Díaz y Romero, propietaria de la finca, autoriza el acceso al yacimiento y ayuda agradablemente a algunas labores logísticas. El doctor Esteban García Viñas ha colaborado en la excavación. Han participado alumnos de la Facultad de Geografía e Historia, miembros asimismo de EvoCultura - Asociación para el estudio del comportamiento humano y la diversidad cultural, que también han colaborado en el dibujo del material arqueológico: Patricia Virino Gabella, David López Carmona, Elena Trujillo Godoy y Araceli Barrera Cruz. Se agradece a los Editores de Munibe Antropologia-Arkeologia el interés en este trabajo; y asimismo a un Editor invitado y a dos revisores, los tres anónimos, por sus comentarios e indicaciones que han dado mayor consistencia y claridad a la versión final del artículo.

\section{BIBLIOGRAFÍA}

Acosta, P., 1987. El neolítico antiguo en el Suroeste español: La Cueva de la Dehesilla (Cádiz). In: Premières Communautés Paysannes en Méditerranée occidentale, Actes du Colloque International du CNRS, Montpellier (1983), 653-659. CNRS, Paris.
Acosta, P., 1995. Las culturas del neolítico y calcolítico en Andalucía Occidental. Espacio, Tiempo y Forma 8, 33-80.

Acosta, P., Pellicer, M., 1990 La Cueva de la Dehesilla (Jerez de la Frontera). Las primeras civilizaciones productoras en Andalucía occidental. CSIC, Jerez de la Frontera.

Amores, F., 2009. Recuerdos del gabinete de dibujo de Pilar Acosta. In: Cruz-Auñón, R., Ferrer, E. (Eds.), Estudios de Prehistoria y Arqueología en Homenaje a Pilar Acosta Martínez, 19-36. Universidad de Sevilla, Sevilla.

Aura, J.E., Jordá, J.F., González, J., Bécares, J., Sanchidrián, J., 1998. Secuencia arqueológica de la Cueva de Nerja: la Sala del Vestíbulo. In: Sanchidrián, J.L., Simón, M.D. (Eds.), Las culturas del Pleistoceno superior en Andalucía, 217-236. Patronato de la Cueva de Nerja, Málaga.

Baena, J., Morgado, A.M., Lozano, J.A., Torres, C., Alcalá, A., Bermúdez, R., Bermúdez, F., Ruiz, F., 2012. Titanes en el Complejo Motillas. La secuencia del Pleistoceno Superior de la cueva del Higueral-Guardia en la bética occidental (Proyecto Kuretes). Menga: Revista de prehistoria de Andalucía 3, 107-117.

Bagolini, B., 1968. Ricerche sulle dimensioni dei manufatti litici prehistorici non ritoccati. Annali dell'Universitá di Ferrara 1(10), Sezione 15, 195-219.

Berger, J.F., Metallinou, G., Guilaine, J., 2014. Vers une révision de la transition méso-néolithique sur le site de Sidari (Corfou, Grèce): nouvelles données géoarchéologiques et radiocarbone, évaluation des processus post-dépositionnels. In: Manen C., Guilaine, J., Perrin, T. (Eds), La trasition néolithique en méditerrannée, 213-232. Archives d’Écologie Préhistorique, Errance, Arles.

Bernabeu, J., Barton, C.M., Pérez, M., 2001. A taphonomic perspective on neolithic beginning: theory, interpretation, and empirical data in the western Mediterranean. Journal of Archaeological Science 28(6), 597-612.

Bernabeu, J., Molina, L., Esquembre, M.A., Ortega, J.R., Boronat, J., 2009. La cerámica impresa mediterránea en el origen del Neolítico de la península Ibérica. In: De Méditerranée et d'ailleurs. Melanges offerts à Jean Guilaine, 83-95. Archives d'Ecologie Pré- historique, Toulouse.

Bernabeu, J., Gómez, O., Molina, L., García, P., 2011. La cerámica neolítica durante el VI milenio AC en el Mediterráneo central peninsular. In: Bernabéu, J., Rojo, M.A, Molina, L. (Guest Eds.), Las primeras producciones cerámicas: el VI milenio cal AC en la Península lbérica. Saguntum PLAV, Extra 12, 153-178.

Bernaldo-Quirós, F., Cabrera, V., Cacho, C., Vega, L.G., 1981. Proyecto de análisis técnico para las industrias líticas. Trabajos de Prehistoria 38, 9-37.

Binder, D., Lanos, P., Angeli, L., Gomart, L., Guilaine, J., Manen, C., Maggi, R., Muntoni, I.M., Panelli, C., Radi, G., Tozzi, C., Arobba, D., Battentier, J., Brandaglia, M., Bouby, L., Briois, F., Carré, A., Delhon, C., Gourichon, L., Marinval, P., Nisbert, R., Rossi, S., Rowley, P., Thiébault, S., 2017. Modelling the earliest north-western dispersal of Mediterranean Impressed Wares: new dates and Bayesian chronological model. Documenta Praehistorica 44, 54-76.

Boessneck, J., 1980. Diferencias osteológicas entre la oveja (Ovis aries linné) y la cabra (Capra hircus linné). In Brothwell, D., Higgs, E., Clark, G. (Eds.), Ciencia en Arqueología, 338366. Fondo de Cultura Económica, Madrid.

Carballo, L.X., Fábregas, R., 1991. Dataciones de carbono 14 para castros del noroeste peninsular. Archivo Español de Arqueología 64, 244-264. 
Caro, A., 1991. Lebrija: La Ciudad y su entorno, I (Prehistoria y Protohistoria). Ayuntamiento de Lebrija y El Monte, Cádiz.

Caro, A., Acosta, P., Escacena, J.L., 1987. Informe sobre la prospección arqueológica con sondeo estratigráfico en el solar de la calle Alcazaba (Lebrija-Sevilla). Anuario Arqueológico de Andalucía 1986(2), 168-174.

Carvalho, A.F., 1998. Talhe da pedra no Neolítico antigo do Maciço Calcário das Serras d'Aire e Candeeiros (Estremadura Portuguesa). Um primeiro modelo tecnológico e tipológico. Colibri, Lisboa.

Carvalho, A.F., Gibaja, J.F., 2005. Talhe da pedra no Neolítico antigo do Maciço Calcário Estremenho (Portugal): matérias-primas, tecnologia e análise funcional. In: Arias, P., Ontañón, R., García-Moncó, C. (Eds.), III Congreso del Neolítico en la Península Ibérica. Monografías del Instituto Internacional de Investigaciones Prehistóricas de Cantabria 1, 373-382. Universidad de Cantabria, Santander.

Cortés, M., Simón, M.D., 2007. La Pileta (Benaoján, Málaga) cien años después. Aportaciones al conocimiento de su secuencia arqueológica. Saguntum 39, 45-64.

Égüez, N., Mallol, C., Martín, D., Camalich, M.D., 2016. Radiometric dates and micromorphological evidence for synchronous domestic activity and sheep penning in a Neolithic cave: Cueva de El Toro (Málaga, Antequera, Spain). Archaeological \& Anthropological Sciences 8(1), 107-123.

Escacena, J.L., 2014. El desembarco de Noé. Sobre la primera neolitización de la paleoensenada bética. In: Parodi, M.J. (Coord.), Ex illo tempore. I Jornadas de Arqueología del Bajo Guadalquivir, 59-129. Cádiz.

Escacena, J.L, García-Rivero, D., En prensa. El neolítico de Lebrija (Sevilla). In: Martínez, G., Afonso, J.A., (Eds.), VI Congreso del Neolítico en la Península Ibérica. Granada.

Fernández, S., Fuentes, N., Carrión, J.S., González, P., Montoya, E., Gil, G., Vera, G., Riquelme, J. A., 2007. The Holocene and upper Pleistocene pollen sequence of Carihuela Cave, southern Spain. Geobios 40(1), 75-90.

Fortea, F.J., Martí, B., 1985. Consideraciones sobre los inicios del Neolítico en el Mediterráneo español. Zephyrus 37-38, 167-199.

Fregel, R., Méndez, F.L., Bokbot, Y., Martín, D., Camalich, M.D., Santana, J., Morales, J., Ávila, M.C., Underhill, P.A., Shapiro, B., Wojcik, G., Rasmussen, M., Soares, A.E.R., Kapp, J., Sockell, A., Rodríguez, F.J., Mikdad, A., Trujillo, A., Bustamante, C.D., 2018. Ancient genomes from North Africa evidence prehistoric migrations to the Maghreb from both the Levant and Europe. Proceedings of the National Academy of Sciences 115(26), 6774-6779

García-Rivero, D., Barrera, J.A., Díaz, F., Borja, C., Álvarez, G., Sancho, F., Escacena, J.L., Pérez, J., En prensa, a. El proyecto 'Cueva de la Dehesilla: Estudio arqueológico y paleoambiental para el conocimiento de la ocupación humana prehistórica de la Sierra de Cádiz': Resultados preliminares de la primera fase - 2015. Anuario Arqueológico de Andalucía 2015.

García-Rivero, D., Barrera, J.A., Díaz, F., Borja, C., Álvarez, G., Sancho, F., Escacena, J.L., Taylor, R., Pérez, J., En prensa, b. El Proyecto Cueva de la Dehesilla (Jerez de la Frontera, Cádiz): primeros resultados tras las intervenciones de 2015. In: Martínez, G., Alfonso, J.A., (Eds.), VI Congreso del Neolítico en la Península Ibérica. Granada.

García-Rivero, D., Taylor, R., Pérez, L.G., Pérez, G., García, E., López, A., Zurro, D., Peña, L., Bernáldez, E., En prensa, c. Andalusi populations at La Dehesilla Cave (Sierra de Cádiz,
Southern Iberia): an interdisciplinary approach to their rural economic systems. Journal of Islamic Archaeology.

García-Borja, P., Aura, J.E., Bernabeu, J., Jordá, J.F., 2010. Nuevas perspectivas sobre la Neolitización en la Cueva de Nerja (Málaga-España): La cerámica de la sala del Vestíbulo. Zephyrus 66, 109-132.

García-Borja, P., Aura, J.E., Jordá, J.F., Salazar, D.C., 2014. La cerámica neolítica de la Cueva de Nerja (Málaga, España): salas del Vestíbulo y la Mina. Archivo de Prehistoria Levantina $30,81-131$.

Giles, F., Gutiérrez, J.M., Santiago, A., Mata, E., 1996. Avance al estudio sobre poblamiento del Paleolítico Superior en la cuenca media y alta del río Guadalete (Cádiz). Boletín del Museo de Cádiz 7, 37-62.

Gómez-Peña, A., Ruiz, E., Pérez, L.G., Guillén, L., 2014. Revisión historiográfica sobre las prospecciones arqueológicas en el término municipal de Trebujena (Cádiz). In: Parodi, M.J., (Coord.), Ex illo tempore. I Jornadas de Arqueología del Bajo Guadalquivir, 251-265. Cádiz.

González, C., Sánchez, M.P., Villafranca, E., 1987. University of Granada. Radiocarbon dates IV. Radiocarbon 29(3), 381-388.

Guerrero-Misa, L.J., 1992. La Sima S-6 del Complejo neolítico de Veredilla (Benaocaz, Cádiz). Spal 1, 79-97.

Guilaine, J., Manen, C., 2002. La ceramica impressa della Francia meridionale. In Fugazzola, M.A., Pessina, A., Tiné, V. (Eds.), Le ceramiche impresse nel Neolitico antico. Italia e Mediterráneo, 37-49. Istituto Poligrafico e Zecca dello Stato, Roma.

Guilaine J., Manen, C., Vigne J.D., 2007 (Eds.). Pont de Roque-Haute. Nouveaux regards sur la Néolithisation de la France méditerranéenne. Archives d'Ecologie Pré- historique, Toulouse.

Gutiérrez-López, J.M., Prieto, M.C., Ruiz, J.A., 1996. Yacimientos neolíticos al aire libre con cardiales el asentamiento de Esperilla (Espera, Cádiz). Propuesta de otro modelo de neolitización para Andalucía occidental. In: Actes I Congrés del Neolític a la Península Ibèrica. Rubricatum 2, 627-638.

Gutiérrez-López, J.M., Reinoso, M.C., Aguilera, L., Santiago, A., 2000. Un balance del Neolítico de las Subbéticas Occidentales al final del Milenio. In: Santiago, A., Martínez, A., Mayoral, J., (Eds.), Actas del I Congreso Andaluz de Espeleología, 151-175. Ayuntamiento de Ronda y Federación Andaluza de Espeleología, Sevilla.

Harris, E.C., 1991. Principios de estratigrafía arqueológica. Crítica, Barcelona.

Jennings, R.P., Giles, F., Barton, R.N.E., Collcutt, S.N., Gale, R., Gleed, C.P., Gutiérrez, J.M., Higham, T.F.G., Parker, A., Price, C., Rhodes, E., Santiago, A., Schwenninger, J.L., Turner, E., 2009. New dates and palaeoenvironmental evidence for the Middle to Upper Palaeolithic occupation of Higueral de Valleja Cave, southern Spain. Quaternary Science Reviews 28, 830-839.

Jordá, J.F., Aura, J.E., 2008. 70 fechas para una cueva: revisión crítica de 70 dataciones C14 del Pleistoceno Superior y Holoceno de la Cueva de Nerja (Málaga, Andalucía, España). Espacio, Tiempo y Forma 1, 239-256.

Juan-Cabanilles, J., 2008. El utillaje de piedra tallada en la Prehistoria reciente valenciana. Aspectos tipológicos, estilísticos y evolutivos. Diputación de Valencia, Valencia.

Laplace, G., 1966. Recherches sur l'origine et l'évolution des complexes leptolithiques. Mélanges d'Archeologie et d'Histoire 4. Ecole Française de Rome, París. 
Lavado, M.L., 1990. Carta arqueológica de la margen izquierda de la desembocadura del Guadalquivir: Sanlúcar (norte) y Trebujena. Anuario Arqueológico de Andalucía 1987(3), 126-133.

Lazarich, M., Richarte, M.J., 2003. El Jadramil (Arcos de la Frontera): estudio arqueológico de un asentamiento agrícola en la campiña gaditana. Ayuntamiento de Arcos de la Frontera, Cádiz.

Martí, F., Sanmartí, E., Viñas, R., 1975. La cueva de «La Dehesilla» en Jerez de la Frontera. In: Ripoll, E., Llongueras, M., (Eds.), XXV Aniversario de los Cursos Internacionales de Prehistoria y Arqueología en Ampurias (1947-1971), 13-47. Diputación Provincial de Barcelona, Barcelona.

Martín-Socas, D., Camalich, M.D., Caro, J.L., Rodríguez, F.J., 2018. The beginning of the Neolithic in Andalusia, Quaternary International 470, 451-471.

Martín-Socas, D., Camalich, M.D., González, P., 2004. La Cueva de El Toro (Sierra de El Torcal-Antequera-Málaga). Un modelo de ocupación ganadera en el territorio andaluz entre el VI y II milenios a.n.e. Consejería de Cultura de la Junta de Andalucía, Sevilla.

Martínez-Fernández, G., Afonso, J.A., 2008. L'evolution des ensembles d'artefacts en pierre taillé pendant la Préhistoire récente au Sud-est de la Péninsule ibérique, In: Dias-Meirinho, M.H., Léa, V., Gernigon, K., Fouéré, P., Briois, F., Bailly, M., (Eds.), Les industries lithiques taillées des IVe et Ille millénaires en Europe Occidentale, 291-308. Hadrianbooks, Oxford.

Mederos, A., 1996. La cronología absoluta de Andalucía Occidental durante la Prehistoria Reciente (6100-850 AC). Spal 5, 45-86.

Medved, I., 2013. Continuity vs. discontinuity. Epipaleolithic and Early Neolithic in the Mediterranen Southeast of the Iberian Peninsula. Tesis doctoral, Universität zu Kölh.

Molina, F., Cámara, J.A., Alfonso, J.A., Gámiz, J., Capel, J., Martínez, G., 2017. Hiatus in an archaeological multilevel site: Los Castillejos in Las Peñas de los Gitanos (Montefrío, Granada), In: Cupitò, M., Vidale, M., Angelini, A., (Eds.), Beyong limits. Studi in onore di Giovanni Leonardi, 91-100. Università degli Studi di Padova, Padova.

Mora-Figueroa, L., 1970. El yacimiento prehistórico de la Cueva de Picado (Cádiz). Trabajos de Prehistoria 27, 279-286.

Mora-Figueroa, L., 1976. El yacimiento prehistórico de la Cueva de Hundidero-Gato, Benaoján (Málaga): I campaña. Noticiario Arqueológico Hispánico 5, 97-106.

Pellicer, M., Acosta, P., 1982. El Neolítico antiguo en Andalucía occidental. In: Le Néolithique Ancien Méditerranéen. Archéologia en Languedoc 7, 49-60. Lattes.

Pellicer, M., Acosta, P. (Coords.), 1997. El Neolítico y Calcolítico de la Cueva de Nerja en el contexto andaluz. Trabajos sobre la Cueva de Nerja 6. Patronato de la Cueva de Nerja, Málaga.

Peña-Chocarro, L., Pérez, G., Morales, J., Vera, J.C., 2013. ...Y llegaron los agricultores: agricultura y recolección en el occidente del Mediterráneo. Menga 4, 15-33.

Perdigones, L., 1987. Carta Arqueológica de Arcos de la Frontera (Cádiz). Memoria de Licenciatura inédita, Universidad de Sevilla.

Pérez-Rodríguez, M., Vijande, E., Cantillo, J.J., 2010. Campos de silos y necrópolis en los entornos de la bahía de Cádiz. Nuevos hallazgos referentes a sociedades tribales. In: Gibaja,
J.F., Carvalho, A.F., (Eds.), Os últimos caçadores-recolectores e as primeiras comunidades productoras do sul da Península Ibérica e do norte de Marrocos, 229-235. Universidade do Algarve. Faro.

Ramos, J., Giles, F., 1996. El dolmen de Alberite: Villamartín: aportaciones a las formas económicas y sociales de las comunidades neolíticas en el noroeste de Cádiz. Universidad de Cádiz y Ayuntamiento de Villamartín, Cádiz.

Reimer, P.J., Bard, E., Bayliss, A., Beck, J.W., Blackwell, P.G., Bronk-Ramsey, C., Grootes, P.M., Guilderson, T.P., Haflidason, H., Hajdas, I., Hatt, C., Heaton, T.J., Hoffman, D.L., Hogg, A.G., Hughen, K.A., Kaiser, K.F., Kromer, B., Manning, S.W., Niu, M., Reimer, R.W., Richards, D.A., Scott, E.M., Southon, J.R., Staff, R.A., Turney, C.S.M. y Van Der Plicht, J., 2013. IntCal13 and Marine13 radiocarbon age calibration curves $0-50,000$ years cal BP. Radiocarbon 55 (4), 1869-1887.

Santiago, A., Gutiérrez, J.M., Giles, F., Pedroche, A., Mendoza, D., Prieto, M.C., 1997. Arte paleolítico en la serranía de Grazalema. La cavidad VR-15. Revista de Arqueología 195, 10-19.

Taylor, R., Pérez, L.G., García, D., 2018. La secuencia arqueológica andalusí (siglos XI-XII) de la Cueva de La Dehesilla (Sierra de Cádiz, España). Arqueología y Territorio Medieval 25, 107-143.

Valdiosera, C., Günther, T., Vera, J.C., Ureña, I., Iriarte, E., Rodríguez, R., Simões, L.G., Martínez, R., Svensson, E.M., Malmström, H., Rodríguez, L., Bermúdez, J.M., Carbonell, E., Alday, A., Hernández, J.A., Götherström, A., Carretero, J.M., Arsuaga, J.L., Smith, C.I., Jakobsson, M., 2018. Four millennia of Iberian biomolecular prehistory illustrate the impact of prehistoric migrations at the far end of Eurasia. Proceedings of the National Academy of Sciences 115(13), 3428-3433.

Vera-Rodríguez, J.C., Gavilán, B., 1999. Organización interna y usos del espacio en la Cueva de Los Murciélagos de Zuheros (Córdoba). In: Bernabeu, J., Orozco, T. (Eds.), Actes del II Congrés del Neolític a la Península Ibérica, Saguntum Extra-2. Universitat de València, Valencia, 229-234.

Vicent, A.M., Muñoz, A.M., 1973. Segunda campaña de excavaciones en la Cueva de los Murciélagos, Zuheros (Córdoba), 1969. Excavaciones Arqueológicas en España 77. Ministerio de Educación, Madrid.

Viñas-Vallverdú, R., 1970. La Cueva de la Dehesilla en Jerez de la Frontera (Cádiz). In: Actas del I Congreso Nacional de Espeleología, 151-153. Barcelona.

Viñas-Vallverdú, R., 1971. Prospecciones espeleo-arqueológicas en la cueva de La Dehesilla, Jerez de la Frontera. Información Arqueológica 6, 176-177.

Zeder, M.A., Lapham, H.A., 2010. Assessing the reliability of criteria used to identify postcranial bones in sheep, Ovis, and goats, Capra. Journal of Archaeological Science 37, 2887-2905.

Zeder, M.A., Pilaar, S.E., 2010. Assessing the reliability of criteria used to identify mandibles and mandibular teeth in sheep, Ovis, and goats, Capra. Journal of Archaeological Science, 37(2), 225-242.

Zilhão, J., 1993. The spread of agro-pastoral economies across Mediterranean Europe: a view from the Far West. Journal of Mediterranean Archaeology 6(1), 5-63 